\title{
On continued fraction expansions of quadratic irrationals in positive characteristic
}

\author{
Frédéric Paulin and Uri Shapira
}

February 1, 2018

\begin{abstract}
Let $R=\mathbb{F}_{q}[Y]$ be the ring of polynomials over a finite field $\mathbb{F}_{q}$, let $\widehat{K}=\mathbb{F}_{q}\left(\left(Y^{-1}\right)\right)$ be the field of formal Laurent series over $\mathbb{F}_{q}$, let $f \in \widehat{K}$ be a quadratic irrational over $\mathbb{F}_{q}(Y)$ and let $P \in R$ be an irreducible polynomial. We study the asymptotic properties of the degrees of the coefficients of the continued fraction expansion of quadratic irrationals such as $P^{n} f$ as $n \rightarrow+\infty$, proving, in sharp contrast with the case of quadratic irrationals in $\mathbb{R}$ over $\mathbb{Q}$ considered in [AS], that they have one such degree very large with respect to the other ones. We use arguments of [BP] giving a relationship with the discrete geodesic flow on the Bruhat-Tits building of $\left(\mathrm{PGL}_{2}, \widehat{K}\right)$ and, with $A$ the diagonal subgroup of $\mathrm{PGL}_{2}(\widehat{K})$, the escape of mass phenomena of [KePS] for $A$-invariant probability measures on the compact $A$-orbits along Hecke rays in the moduli space $\mathrm{PGL}_{2}(R) \backslash \mathrm{PGL}_{2}(\widehat{K}){ }^{1}$
\end{abstract}

\section{Introduction}

This paper gives a positive characteristic analogue (with a surprisingly different outcome) of results of Aka-Shapira [AS]. They studied the statistics of the period of the continued fraction expansion of arithmetically constructed sequences of quadratic irrational real numbers. For instance, given $\alpha \in \mathbb{R}$ a quadratic irrational over $\mathbb{Q}$ and $p \in \mathbb{Z}$ a prime, they proved that the equiprobability measures on the periodic part of the orbit of the fractional part of $p^{n} \alpha$ under the Gauss map weak-star converges towards the Gauss-measure on $[0,1]$ as $n \rightarrow+\infty$. Using the dynamical approach of [KePS] and giving the arithmetic applications announced there, we will prove that in positive characteristic on the contrary, the coefficients of the continued fraction expansion along similar sequences of quadratic irrationals have a very irregular distribution (see in particular Theorem 2), and that the proportion of high degree coefficients might be positive.

Let us give a more precise description of our results. Let $\mathbb{F}_{q}$ be a finite field of order a positive power $q$ of a prime $p$ different from 2, and let $K=\mathbb{F}_{q}(Y)$ be the field of rational functions in one variable $Y$ over $\mathbb{F}_{q}$. Let $R=\mathbb{F}_{q}[Y]$ be the ring of polynomials in $Y$ over $\mathbb{F}_{q}$, let $\widehat{K}=\mathbb{F}_{q}\left(\left(Y^{-1}\right)\right)$ be the non-Archimedean local field of formal Laurent series in $Y^{-1}$ over $\mathbb{F}_{q}$, let $\mathscr{O}=\mathbb{F}_{q}\left[\left[Y^{-1}\right]\right]$ be the local ring of $\widehat{K}$ (consisting of formal power series in $Y^{-1}$ over $\mathbb{F}_{q}$ ), and let $\mathscr{M}=Y^{-1} \mathscr{O}$ be the maximal ideal of $\mathscr{O}$.

\footnotetext{
${ }^{1}$ Keywords: quadratic irrational, continued fraction expansion, positive characteristic, Artin map, Hecke tree, Bruhat-Tits tree. AMS codes: 11J70, 20E08, 20G25, 37A17, 22F30.
} 
Any element $f \in \widehat{K}$ may be uniquely written $f=[f]+\{f\}$ with $[f] \in R$ (called the integral part of $f$ ) and $\{f\} \in \mathscr{M}$ (called the fractional part of $f$ ). The Artin map $\Psi: \mathscr{M}-\{0\} \rightarrow \mathscr{M}$ is defined by $f \mapsto\left\{\frac{1}{f}\right\}$. Any $f \in \widehat{K}$ which is irrational (that is, not in $K$ ) has a unique continued fraction expansion

$$
f=a_{0}+\frac{1}{a_{1}+\frac{1}{a_{2}+\frac{1}{a_{3}+\cdots}}},
$$

with $a_{0}=a_{0}(f)=[f] \in R$ and $a_{n}=a_{n}(f)=\left[\frac{1}{\Psi^{n-1}\left(f-a_{0}\right)}\right]$ a non constant polynomial for $n \geq 1$ (called the coefficients of the continued fraction expansion of $f$ ).

Let $Q I=\{f \in \widehat{K}:[K(f): K]=2\}$ be the set of quadratic irrationals over $K$ in $\widehat{K}$. Given $f \in \widehat{K}-K$, it is well known that $f \in Q I$ if and only if the continued fraction expansion of $f$ is eventually periodic. Note that the projective action (denoted by $\cdot$ ) of $\mathrm{PGL}_{2}(R)$ on $\mathbb{P}_{1}(\widehat{K})=\widehat{K} \cup\{\infty\}$ preserves $Q I$, keeping the periodic part of the continued fraction expansions unchanged (up to cyclic permutation and invertible elements, see [BN]), and that for all $x \in K$ and $f \in Q I$, we have $x f \in Q I$. We refer for instance to the surveys [Las, Sch] for background on the above notions.

For every $f \in Q I$, we denote by $\ell_{f}$ the length of the period of the eventually periodic continued fraction expansion of $f$, and by $d_{f, 1}, \ldots, d_{f, \ell_{f}} \in \mathbb{N}-\{0\}$ the degrees of the polynomial coefficients appearing in this period. For $c \in] 0,1]$, we say that a sequence $\left(f_{n}\right)_{n \in \mathbb{N}}$ in $Q I$ has $c$-degree-escaping continued fraction expansions if for every $N \in \mathbb{N}$,

$$
\liminf _{n \rightarrow+\infty} \frac{\max _{i=1, \ldots, \ell_{f_{n}}} d_{f_{n}, i}-N}{\sum_{i=1}^{\ell_{f_{n}}} d_{f_{n}, i}} \geq c .
$$

Theorem 1 Let $f \in Q I$.

(1) For every irreducible polynomial $P \in R$, there exists $c=c_{f, P}>0$ such that the sequence $\left(P^{n} f\right)_{n \in \mathbb{N}}$ has c-degree-escaping continued fraction expansions.

(2) Let $\gamma \in \mathrm{GL}_{2}(R)$ projectively fixing $f$ whose discriminant $P=(\operatorname{tr} \gamma)^{2}-4 \operatorname{det} \gamma$ is irreducible over $\mathbb{F}_{q}$. Then for all $N \in \mathbb{N}$,

$$
\limsup _{n \rightarrow+\infty} \frac{\max _{i=1, \ldots, \ell_{P^{n}}} d_{P^{n} f, i}-N}{\sum_{i=1}^{\ell_{P^{n}}} d_{P^{n} f, i}}=1 .
$$

(3) For every irreducible polynomial $P \in R$, there exists $c=c_{f, P}>0$ such that for uncountably many sequences $\left(\gamma_{n}^{\prime}\right)_{n \in \mathbb{N}}$ in $\operatorname{PGL}\left(\mathbb{F}_{q}\left[Y, \frac{1}{P}\right]\right)$, the sequence $\left(\gamma_{n}^{\prime} \cdot f\right)_{n \in \mathbb{N}}$ has c-degree-escaping continued fraction expansions.

Given $f \in Q I$ and an irreducible ${ }^{2}$ polynomial $P \in R$, the study of the properties of the continued fraction expansion of $\left(P^{n} f\right)_{n \in \mathbb{N}}$ is interesting in particular by the analogy with the case of quadratic irrationals in $\mathbb{R}$ over $\mathbb{Q}$, and the above results are strikingly different

\footnotetext{
${ }^{2}$ This irreducibility assumption in not essential, it only simplifies the exposition so that we don't have to work with several completions simultaneously.
} 
from the ones obtained in $[\mathrm{AS}]$. Indeed, given $f \in \mathbb{R}$ a quadratic irrationnal over $\mathbb{Q}$ whose continued fraction expansion $\left(a_{n}(f)\right)_{n \in \mathbb{N}}$ is periodic after the time $k_{f} \geq 1$ with period $\ell_{f}$, as a consequence of the much stronger results in [AS], we have, for every positive prime integer $p$,

$$
\lim _{n \rightarrow+\infty} \frac{\max _{i=k_{f}, \ldots, k_{f}+\ell_{p^{n}}-1} \log a_{i}\left(p^{n} f\right)}{\sum_{i=k_{f}}^{k_{f}+\ell_{p} n_{f}-1} \log a_{i}\left(p^{n} f\right)}=0 .
$$

Theorem 4.5 of [dMT] has, using [dMT, Lem. 4.2], the following consequence : If $\left(a_{n, k}\right)_{k \in \mathbb{N}}$ is the continued fraction expansion of $P_{n} f$ for all $n \in \mathbb{N}$, where $\left(P_{n}\right)_{n \in \mathbb{N}}$ is a sequence in $\mathbb{F}_{q}[Y]$ with $P_{n}$ dividing $P_{n+1}$ and $P_{n+1} / P_{n}$ having bounded degree, then $\sup _{n, k \in \mathbb{N}-\{0\}} \operatorname{deg} a_{n, k}=+\infty$. Assertion (1) of Theorem 1 implies a much stronger result for the sequence $\left(P_{n}=P^{n}\right)_{n \in \mathbb{N}}$ of powers of any irreducible polynomial $P \in R$.

Assertion (2) of Theorem 1, with the explicit example given after [KePS, Lem. 13], implies for instance that if $p=q=3, P=Y^{2}+4$ and $f=\frac{Y-\sqrt{Y^{2}+4}}{2}$ so that the Galois conjugate $f^{\sigma}$ of $f$ is the well-known continued fraction expansion $f^{\sigma}=Y+\frac{1}{Y+\frac{1}{Y+\cdots}}$, then Equation (1) holds.

The main point in the proof of Theorem 1 is to relate the orbits of quadratic irrationals under the Artin map to the compact orbits of the diagonal subgroup $A$ of $G=\operatorname{PGL}_{2}(\widehat{K})$ on the moduli space $X$ of $R$-lattices (up to homotheties) in $\widehat{K} \times \widehat{K}$. More precisely (forgetting about measure zero subsets in this introduction, see Section 2 for exact statements), let $\Gamma=\mathrm{PGL}_{2}(R)$ be Nagao's lattice (see [Nag, Wei]) so that $X$ is the homogeneous space $\Gamma \backslash G$ endowed with the action of $A$ by translations on the right. We construct, using [BP] and [EW, §9.6] as inspiration, a natural cross-section $C$ for the action of $A$ on $X$ and a natural map $\Theta_{2}: C \rightarrow \mathscr{M}$ such that if $T: C \rightarrow C$ is the first return map in $C$ of the orbits under $A$, then we have a commutative diagram

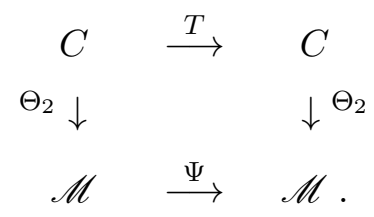

Given a quadratic irrational $f$, assuming for simplicity that $f \in \mathscr{M}$ and that its Galois conjugate does not belong to $\mathscr{O}$, we construct in Lemma 6 an explicit element $x_{f} \in C$ whose $A$-orbit in $X$ is compact, such that the image by $\Theta_{2}$ of the intersection with $C$ of the orbit of $x_{f}$ by right translations under the semigroup $A_{+}=\left\{\left[\begin{array}{ll}1 & 0 \\ 0 & f\end{array}\right]: f \in \widehat{K}-\mathscr{O}\right\}$, is equal to the orbit of $f$ under the iterations of $\Psi$.

We then apply in Section 3 the results of [KePS] on the escape of mass of the $A$-invariant probability measures on the compact $A$-orbits in $X$ varying along rays in the Hecke trees of elements of $X$ associated to a given irreducible polynomial in $R$.

We conclude this introduction by stating a distribution result for the orbits under the Artin maps of arithmeticaly defined families of quadratic irrationals, again in sharp contrast with the ones obtained in [AS], and again using the above-mentionned results of $[\mathrm{KePS}]$. For every $f \in Q I$, let $\nu_{f}$ be the equiprobability on the periodic part of the 
$\Psi$-orbit of $\{f\}$. Given nice sequences $\left(f_{n}\right)_{n \in \mathbb{N}}$ in $Q I$, it is interesting to study the weak-star accumulation points of the equiprobabilities $\nu_{f_{n}}$ as $n \rightarrow+\infty$.

Theorem 2 Let $f \in Q I$ and $P \in R$ be an irreducible polynomial. For uncountably many sequences $\left(\gamma_{n}^{\prime \prime}\right)_{n \in \mathbb{N}}$ in $\mathrm{PGL}_{2}(R)$, for every $f^{\prime} \in Q I$, there exists $c>0$ and a weak-star accumulation point $\theta$ of the equiprobabilities $\nu_{P^{n}} \gamma_{n}^{\prime \prime} \cdot f$ such that $\theta \geq c \nu_{f^{\prime}}$.

In particular, $\theta$ is not absolutely continuous with respect to the Haar measure of $\mathscr{M}$, which is the analog for the Artin map $\Psi$ of the Gauss measure for the Gauss map. Thus this proves that there are many Hecke-type sequences $\left(P^{n_{k}} \gamma_{n_{k}} \cdot f\right)_{k \in \mathbb{N}}$ of quadratic irrationals whose continued fraction expansions not only have coefficients with degree almost equal to the sum of the degrees on the period, but also have a positive proportion of coefficients which are constant (say equal to $Y$, taking for instance $f^{\prime}=Y+\frac{1}{Y+\frac{1}{Y+\cdots}}$.

Acknowledgements: A first version of this work was partially supported by the NSF grants DMS-1440140, while the first author was in residence at the MSRI, Berkeley CA, during the Fall 2016 semester. The first author would like to thank the Isaac Newton Institute for Mathematical Sciences, Cambridge, for support and hospitality during the programme "Non-positive curvature group actions and cohomology" where work on this paper was completed. This work was supported by EPSRC grant no EP/K032208/1. The second author is supported by ISF grants $357 / 13$ and $871 / 17$.

\section{A cross-section for the orbits of the diagonal group}

In this first section, we give an explicit correspondence between the orbits in the moduli space $\mathrm{PGL}_{2}(\Gamma) \backslash \mathrm{PGL}_{2}(\widehat{K})$ under the diagonal subgroup and the orbits in $\mathscr{M}$ under the Artin map $\Psi$. We start by recalling some notation and definitions from [Ser, Pau, BP, KePS].

We denote by $v_{\infty}: K \rightarrow \mathbb{Z} \cup\{+\infty\}$ the (normalized, discrete) valuation on $K$ such that $v_{\infty}(P)=-\operatorname{deg} P$ for all $P \in R-\{0\}$ (and $v_{\infty}(0)=+\infty$ ), and again by $v_{\infty}: \widehat{K} \rightarrow \mathbb{Z} \cup\{+\infty\}$ its continuous extension to $\widehat{K}$ for the absolute value

$$
|x|_{\infty}=q^{-v_{\infty}(x)}
$$

The convergence of the continued fraction expansions takes place in $\widehat{K}$ endowed with $|\cdot|_{\infty}$.

Let $G$ be the locally compact group $\mathrm{PGL}_{2}(\widehat{K})$, and let $\Gamma=\operatorname{PGL}_{2}(R)$, which is a nonuniform lattice in $G$. Let $A$ be the image in $G$ of the diagonal subgroup of $\mathrm{GL}_{2}(\widehat{K})$. We denote by $\underline{A}(\mathscr{O})$ the image in $G$ of the group of diagonal matrices with diagonal coefficients in $\mathscr{O}^{\times}$. We denote by $\left[\begin{array}{ll}a & b \\ c & d\end{array}\right]$ the image in $G$ of $\left(\begin{array}{ll}a & b \\ c & d\end{array}\right) \in \mathrm{GL}_{2}(\widehat{K})$, and by $\alpha_{0}: \widehat{K}^{\times} \rightarrow A$ the group isomorphism $t \mapsto\left[\begin{array}{ll}1 & 0 \\ 0 & t\end{array}\right]$. From now on, we consider $\mathfrak{a}_{0}=\alpha_{0}(Y)$.

Let $X=\Gamma \backslash G$. The map $\Gamma g \mapsto g^{-1}[R \times R]$ identifies $X$ with the space of homothety classes of $R$-lattices in $\widehat{K} \times \widehat{K}$ (that is, of rank 2 free $R$-submodules spanning the vector plane $\widehat{K} \times \widehat{K}$ over $\widehat{K}$ ). A point $x \in X$ is called $A$-periodic if its orbit by right translations 
under $A$ is compact. This orbit $x A$ then carries a unique $A$-invariant probability measure, denoted by $\mu_{x}$.

We denote by $\mathbb{P}_{1}(\widehat{K})^{(3)}$ the space of triples of pairwise distinct points in $\mathbb{P}_{1}(\widehat{K})$, and by $\mathbb{P}_{1}(\widehat{K})^{(3)}$ its intersection with $(\widehat{K}-K)^{2} \times \mathbb{P}_{1}(\widehat{K})$. Recall that the diagonal action of $G=\mathrm{PGL}_{2}(\widehat{K})$ on $\mathbb{P}_{1}(\widehat{K})^{(3)}$ is simply transitive, so that $G$ identifies with $\mathbb{P}_{1}(\widehat{K})^{(3)}$ by the map $g \mapsto g(\infty, 0,1)$ and $X$ identifies with $\Gamma \backslash \mathbb{P}_{1}(\widehat{K})^{(3)}$ by the map $\Gamma g \mapsto \Gamma g(\infty, 0,1)$. We denote by $G^{\natural}$ the dense measurable subset of $G$ corresponding to $\mathbb{P}_{1}(\widehat{K})^{(3)}$. It has full measure for any Haar measure on $G$. It is $\Gamma$-invariant on the left, since the projective action of $\Gamma$ preserves $\mathbb{P}_{1}(\widehat{K})-\mathbb{P}_{1}(K)=\widehat{K}-K$. It is $A$-invariant on the right, since the projective action of $A$ preserves $\infty$ and 0 . We denote by $X^{\natural}$ the dense measurable subset of $X$ corresponding to $\Gamma \backslash \mathbb{P}_{1}(\widehat{K})^{(3)}$, which is $A$-invariant on the right and has full measure for the $G$-invariant probability measure $m_{X}$ on $X$. We also define

$$
\mathscr{M}^{\natural}=\mathscr{M}-(\mathscr{M} \cap K) \text { and }\left({ }^{c} \mathscr{O}\right)^{\natural}=\widehat{K}-(\mathscr{O} \cup K),
$$

the subsets of irrational points in $\mathscr{M}$ and ${ }^{c} \mathscr{O}$ respectively.

Let us recall the minimal necessary background on the Bruhat-Tits tree $\mathbb{T}$ of $\left(\mathrm{PGL}_{2}, \widehat{K}\right)$ from [Ser] or [KePS, §2.3]. It is a regular tree of degree $q+1$, endowed with an action of $G$ by automorphisms (without inversions). Its (discrete) set of vertices $V \mathbb{T}$ is the set of homothety classes (under $\widehat{K}^{\times}$) of $\mathscr{O}$-lattices in $\widehat{K} \times \widehat{K}$, endowed with the maximal distance such that the distance between the endpoints of any edge is 1 .

We denote by $E \mathbb{T}$ its set of edges, by $o: E \mathbb{T} \rightarrow V \mathbb{T}$ the origin map of edges, and by $*=[\mathscr{O} \times \mathscr{O}]$ its standard base point, whose stabilizer in $G$ is the maximal compact subgroup $\mathrm{PGL}_{2}(\mathscr{O})$ of $G$, so that the map $g \mapsto g *$ induces an identification between $G / \mathrm{PGL}_{2}(\mathscr{O})$ and $V \mathbb{T}$.

The boundary at infinity $\partial_{\infty} \mathbb{T}$ of $\mathbb{T}$ identifies ( $G$-equivariantly) with the projective line $\mathbb{P}_{1}(\widehat{K})$ over $\widehat{K}$, which itself identifies with $\widehat{K} \cup\{\infty\}$ using the map $[x: y] \mapsto x y^{-1}$. We endow it with the unique probability measure $\mu_{\mathrm{Hau}}$ invariant under the compact group $\operatorname{PGL}_{2}(\mathscr{O})$. We denote by $g \cdot \xi$ the projective action of $g \in G$ on $\xi \in \mathbb{P}_{1}(\widehat{K})=\partial_{\infty} \mathbb{T}$.

We say that an edge $e$ of $\mathbb{T}$ points towards a point at infinity $\xi \in \partial_{\infty} \mathbb{T}$ if its terminus $t(e)$ belongs to the geodesic ray starting from its origin $o(e)$, with point at infinity $\xi$.

We denote by $\mathscr{G} \mathbb{T}$ the space of geodesic lines in $\mathbb{T}$ (that is, the set of isometric maps $\ell: \mathbb{Z} \rightarrow V \mathbb{T}$ endowed with the compact-open topology), and by $\ell_{*} \in \mathscr{G} \mathbb{T}$ the unique geodesic line with $\ell_{*}(-\infty)=\infty, \ell_{*}(+\infty)=0$, and $\ell_{*}(0)=*$, so that $\ell_{*}(n)=\mathfrak{a}_{0}^{n} *$ for every $n \in \mathbb{Z}$. We denote by $\left(\phi_{n}\right)_{n \in \mathbb{Z}}$ the (discrete time) geodesic flow on $\mathscr{G} \mathbb{T}$, where $\phi_{n} \ell: k \mapsto \ell(k+n)$ for all $n \in \mathbb{Z}$ and $\ell \in \mathscr{G} \mathbb{T}$, as well as its quotient flow on $\Gamma \backslash \mathscr{G} \mathbb{T}$. The stabilizer of $\ell_{*}$ for the transitive action $(g, \ell) \mapsto\{g \ell: k \mapsto g \ell(k)\}$ of $G$ on $\mathscr{G} \mathbb{T}$ is exactly $\underline{A}(\mathscr{O})$. Hence the map $g \mapsto g \ell_{*}$ induces a homeomorphism from $G / \underline{A}(\mathscr{O})$ to $\mathscr{G} \mathbb{T}$. We have the following crucial property relating the right action of $A$ on $X=\Gamma \backslash G$ and the geodesic flow on $\mathscr{G} \mathbb{T}$ : For all $g \in G$ and $n \in \mathbb{Z}$,

$$
\phi_{n}\left(g \ell_{*}\right)=g \mathfrak{a}_{0}^{n} \ell_{*}
$$

Recall that the Buseman function of $\mathbb{T}$ is the map $\beta: \partial_{\infty} \mathbb{T} \times V \mathbb{T} \times V \mathbb{T} \rightarrow \mathbb{R}$ defined by

$$
\beta:(\xi, x, y) \mapsto \beta_{\xi}(x, y)=d(x, p)-d(y, p)
$$

for any point $p$ close enough to $\xi$, and in particular the point $p \in V \mathbb{T}$ such that the geodesic rays $[x, \xi[$ and $[y, \xi[$ with origin respectively $x$ and $y$, with point at infinity $\xi$, 
intersect exactly in the geodesic ray $[p, \xi[$. A (closed) horoball in $\mathbb{T}$ centered at a point at infinity $\xi \in \partial_{\infty} \mathbb{T}$ is a sublevel set $\left\{x \in V \mathbb{T}: \beta_{\xi}\left(x, x_{0}\right) \leq 0\right\}$ for some $x_{0} \in V \mathbb{T}$.

We denote (see for instance [Pau, $\S 6.2]$ ) by $\left(\mathscr{H}_{\xi}\right)_{\xi \in \mathbb{P}_{1}(K)}$ the $\Gamma$-invariant family of maximal horoballs in $\mathbb{T}$ with pairwise disjoint interiors such that the point at infinity of $\mathscr{H}_{\xi}$ is $\xi$. If $\mathscr{H}_{\infty}=\left\{x \in V \mathbb{T}: \beta_{\infty}(x, *) \leq 0\right\}$ is the horoball centered at $\infty$ whose boundary contains $*$, then $\mathscr{H}_{\xi}=\gamma \mathscr{H}_{\infty}$ for every $\gamma \in \Gamma$ such that $\gamma \cdot \infty=\xi$. Note that

$$
\Gamma *=\bigcup_{\xi \in \mathbb{P}_{1}(K)} \partial \mathscr{H}_{\xi}=V \mathbb{T}-\bigcup_{\xi \in \mathbb{P}_{1}(K)} \stackrel{\circ}{\mathscr{H}_{\xi}} .
$$

For instance (see loc.cit.), the horoballs in this family whose boundary contains the base point $*$ are the one centered at the elements in $\mathbb{P}_{1}\left(\mathbb{F}_{q}\right)=\mathbb{F}_{q} \cup\{\infty\}$, and $\mathscr{M}$ (respectively ${ }^{c} \mathscr{O}$, and $\left.\mathscr{O}^{\times}\right)$is the set of points at infinity of the geodesic rays starting from $*$ whose first edge lies in $\mathscr{H}_{0}$ (respectively in $\mathscr{H}_{\infty}$, and neither in $\mathscr{H}_{0}$ nor in $\mathscr{H}_{\infty}$ ).

Let

$$
\begin{aligned}
\widetilde{C} & =\left\{g \in G^{\natural}: g \cdot \infty \in{ }^{c} \mathscr{O}, g \cdot 0 \in \mathscr{M}, g \cdot 1=1\right\} \\
& =\left\{g \in G: g \cdot \infty \in\left({ }^{c} \mathscr{O}\right)^{\natural}, g \cdot 0 \in \mathscr{M}^{\natural}, g \cdot 1=1\right\} .
\end{aligned}
$$

As we shall see in the proof below, we actually have $\widetilde{C} \subset \operatorname{PGL}_{2}(\mathscr{O})$ and if $g \mapsto \bar{g}$ is the group morphism $\mathrm{PGL}_{2}(\mathscr{O}) \rightarrow \mathrm{PGL}_{2}(\mathscr{O} / \mathscr{M})=\mathrm{PGL}_{2}\left(\mathbb{F}_{q}\right)$ of reduction modulo $\mathscr{M}$, then $\bar{g}=$ id for every $g \in \widetilde{C}$.

Let $C=\Gamma \widetilde{C}$ be the image of $\widetilde{C}$ in $X^{\natural}=\Gamma \backslash G^{\natural}$ by the canonical projection. We will in particular prove in the next result that $C$ is a natural cross-section for the right action of $A$ : Every orbit of $A$ in $X^{\natural}$ meets $C$ in an infinite countable subset. ${ }^{3}$ As in [BP, Theo. 3.7], let

$$
\widetilde{\Psi}:\left({ }^{c} \mathscr{O}\right)^{\natural} \times \mathscr{M}^{\natural} \rightarrow\left({ }^{c} \mathscr{O}\right)^{\natural} \times \mathscr{M}^{\natural}
$$

be the map defined by

$$
\left(\xi_{-}, \xi_{+}\right) \mapsto\left(\frac{1}{\xi_{-}}-\left[\frac{1}{\xi_{+}}\right], \Psi\left(\xi_{+}\right)\right)
$$

Theorem 3 (1) The subset $C$ of $X^{\natural}$ is closed in $X^{\natural}$, and the restriction to $\widetilde{C}$ of the canonical projection $G \rightarrow X=\Gamma \backslash G$ is a homeomorphism from $\widetilde{C}$ to $C$.

(2) The map $\widetilde{\Theta}: g \mapsto(g \cdot \infty, g \cdot 0)$ from $\widetilde{C}$ to $\mathbb{P}_{1}(\widehat{K})^{2}$ induces a homeomorphism

$$
\Theta: C \rightarrow\left({ }^{c} \mathscr{O}\right)^{\natural} \times \mathscr{M}^{\natural} .
$$

(3) Every A-orbit in $X^{\natural}$ meets $C$.

(4) For every $x \in C$, there exist unique sequences $\left(\alpha_{k}=\alpha_{k}(x)\right)_{k \in \mathbb{Z}}$ in $\underline{A}(\mathscr{O})$ and $\left(t_{k}=\right.$ $\left.t_{k}(x)\right)_{k \in \mathbb{Z}}$ in $2 \mathbb{Z}$ with $\alpha_{0}=\mathrm{id}, t_{0}=0$ and $t_{k}<t_{k+1}$ for all $k \in \mathbb{Z}$ such that

$$
x A \cap C=\left\{x \alpha_{k} \mathfrak{a}_{0}{ }^{t_{k}}: k \in \mathbb{Z}\right\} .
$$

We denote by $T: C \rightarrow C$ the map $x \mapsto x \alpha_{1} \mathfrak{a}_{0}{ }^{t_{1}}$.

\footnotetext{
${ }^{3}$ This follows from Assertion (3) and Assertion (4) below.
} 
(5) The following diagram is commutative

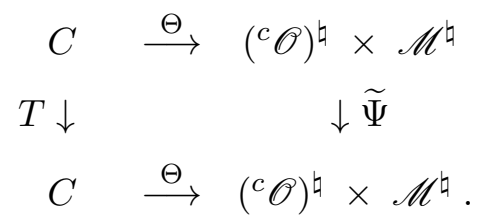

We call $C$ the cross-section for the action of $A$ on $X^{\natural}$. We say that $t_{1}(x)$ is the first return time in $C$ of the orbit of $x \in C$ under $A$, and we call $T$ the first return map of the orbits under $A$ in the cross-section $C$.

Note that $T$ is a homeomorphism, with $t_{m}\left(T^{n} x\right)=t_{m+n}(x)-t_{n}(x)$ and $\alpha_{m}\left(T^{n} x\right)=$ $\alpha_{m+n}(x) \alpha_{n}(x)^{-1}$ for all $m, n \in \mathbb{Z}$.

The commutativity of the diagram in Assertion (5) is inspired by, and closely related to, the commutativity of the diagram in [BP, Theo. 3.7].

Proof. (1) The closure $\overline{\widetilde{C}}$ of $\widetilde{C}$ in $G$ is $\left\{g \in G: g \cdot \infty \in{ }^{c} \mathscr{O}, g \cdot 0 \in \mathscr{M}, g \cdot 1=1\right\}$, which is closed since the conditions on $g \cdot \infty, g \cdot 0$ and $g \cdot 1$ are closed. By the description of the geodesic rays in $\mathbb{T}$ starting from $*$, any geodesic line $\ell$ with endpoints $\ell(-\infty) \in{ }^{c} \mathscr{O}$ and $\ell(+\infty) \in \mathscr{M}$ passes through the base point $*$, and the closest point projection of $1 \in \mathbb{P}_{1}(\widehat{K})=\partial_{\infty} \mathbb{T}$ on $\ell$ is equal to $*$. Hence if $g \in \widetilde{C}$, since $g$ commutes with the closest point projections on geodesic lines, we have $g *=*$. Therefore $g \in \overline{\widetilde{C}}$ is contained in the stabilizer $\mathrm{PGL}_{2}(\mathscr{O})$ of $*$ in $G$, which is compact. Hence $\widetilde{\widetilde{C}}$ is compact and so is its image $\Gamma \overline{\widetilde{C}}$ in $X=\Gamma \backslash G$. Thus $C=X^{\natural} \cap \Gamma \overline{\widetilde{C}}$ is closed in $X^{\sharp}$.

Let us prove that the canonical projection $G \rightarrow X$ is injective on $\widetilde{\widetilde{C}}$. Since its image in $X$ is $\Gamma \overline{\widetilde{C}}$, and since $\overline{\widetilde{C}}$ is compact and $X$ is Hausdorff, this proves that the orbit map $\overline{\widetilde{C}} \rightarrow \Gamma \overline{\widetilde{C}}$ is a homeomorphism, and so is $\widetilde{C} \rightarrow C$ by restriction.

Let us denote by $g \mapsto \bar{g}$ the group morphism $\mathrm{PGL}_{2}(\mathscr{O}) \rightarrow \mathrm{PGL}_{2}(\mathscr{O} / \mathscr{M})=\operatorname{PGL}_{2}\left(\mathbb{F}_{q}\right)$ of reduction of coefficients modulo $\mathscr{M}$, which is the identity map on $\mathrm{PGL}_{2}\left(\mathbb{F}_{q}\right)$. Recall (see for instance [Ser]) that we have an identification between the link

$$
l k(*)=\{e \in E \mathbb{T}: o(e)=*\}
$$

of the vertex $*$ in $\mathbb{T}$ and the finite projective line $\mathbb{P}_{1}\left(\mathbb{F}_{q}\right)=\mathbb{F}_{q} \cup\{\infty\}$ which is equivariant under the reduction morphism $\mathrm{PGL}_{2}(\mathscr{O}) \rightarrow \mathrm{PGL}_{2}\left(\mathbb{F}_{q}\right)$. For every $\gamma \in \Gamma$, if there exists $g, g^{\prime} \in \overline{\widetilde{C}}$ such that $\gamma g=g^{\prime}$, then since $\mathscr{O} \cap R=\mathbb{F}_{q}$, we have

$$
\gamma=g^{\prime} g^{-1} \in \mathrm{PGL}_{2}(\mathscr{O}) \cap \Gamma=\mathrm{PGL}_{2}\left(\mathbb{F}_{q}\right) .
$$

Furthermore, $\gamma$ fixes the edges with origin $*$ pointing towards $\infty, 0$ and 1 respectively, hence $\gamma$ is an element of $\mathrm{PGL}_{2}\left(\mathbb{F}_{q}\right)$ fixing the points $\infty, 0$ and 1 in $\mathbb{P}_{1}\left(\mathbb{F}_{q}\right)$. Since $\mathrm{PGL}_{2}\left(\mathbb{F}_{q}\right)$ acts simply transitively on the set of triples of pairwise distinct elements of $\mathbb{P}_{1}\left(\mathbb{F}_{q}\right)$, we have $\gamma=\mathrm{id}$. Therefore the canonical projection $G \rightarrow \Gamma \backslash G$ is indeed injective on $\widetilde{\widetilde{C}}$. This completes the proof of Assertion (1).

(2) The map from $G$ to $\mathbb{P}_{1}(\widehat{K})^{(3)}$ defined by $g \mapsto(g \cdot \infty, g \cdot 0, g \cdot 1)$ being a homeomorphism, the map from $\overline{\widetilde{C}}$ to $\mathscr{M} \times{ }^{c} \mathscr{O}$ defined by $g \mapsto(g \cdot \infty, g \cdot 0)$ is a homeomorphism. Hence by 
restriction, the map from $\widetilde{C}$ to $\mathscr{M}^{\natural} \times\left({ }^{c} \mathscr{O}\right)^{\natural}$ defined by $g \mapsto(g \cdot \infty, g \cdot 0)$ is a homeomorphism. Assertion (2) then follows from Assertion (1), by composition.

(3) Since $A$ is abelian, the direct product group $\Gamma \times A$ acts on $G$ by $((\gamma, a), g) \mapsto \gamma g a$ for all $\gamma \in \Gamma, g \in G, a \in A$, and this action preserves $G^{\natural}$. Let us prove that every $(\Gamma \times A)$-orbit in $G^{\natural}$ meets $\widetilde{C}$. By taking quotients on the left modulo $\Gamma$, this proves that every $A$-orbit in $X^{\natural}$ meets $C$.

Let us fix $g \in G^{\natural}$. Let us prove that $\Gamma g A$ meets $\widetilde{C}$. Since the family of horoballs $\left(\mathscr{H}_{\xi}\right)_{\xi \in \mathbb{P}_{1}(K)}$ covers $\mathbb{T}$, since its elements have pairwise disjoint interiors, since the boundary of $\mathscr{H}_{\xi}$ is contained in $\Gamma *$ for every $\xi \in \mathbb{P}_{1}(K)$, and since no complete geodesic line is contained in a horoball, the geodesic line $g \ell_{*}$ meets $\Gamma *$. Hence, using Equation (2), there exists $n \in \mathbb{Z}$ such that $g \mathfrak{a}_{0}^{n} *=g \ell_{*}(n) \in \Gamma *$. We may hence assume, up to multiplying $g$ on the right by an element of $\mathfrak{a}_{0}^{\mathbb{Z}} \subset A$, that $g * \in \Gamma *$.

Up to multiplying $g$ on the left by an element of $\Gamma$, we may hence assume that $g *=$ *. Since the link $l k(*)$ of $*$ identifies with the projective line $\mathbb{P}_{1}\left(\mathbb{F}_{q}\right)$, since $\mathrm{PGL}_{2}\left(\mathbb{F}_{q}\right)$ is contained in $\Gamma$ and acts transitively on the ordered pairs of distinct elements of $\mathbb{P}_{1}\left(\mathbb{F}_{q}\right)$, we may assume, up to multiplying $g$ on the left by an element of $\Gamma$, that the edge $e_{\infty}$ with origin $*$ pointing towards $\infty$ also points towards $g \cdot \infty$ and that the edge $e_{0}$ with origin $*$ pointing towards 0 also points towards $g \cdot 0$.

By for instance [Pau] and as recalled before the definition of $\widetilde{C}$, a geodesic ray starting from $*$ has its point at infinity in respectively ${ }^{c} \mathscr{O}$ or $\mathscr{M}$ if and only if its first edge is $e_{\infty}$ or $e_{0}$. In particular, $g \cdot \infty \in\left({ }^{c} \mathscr{O}\right)^{\natural}$ and $g \cdot 0 \in \mathscr{M}^{\natural}$.

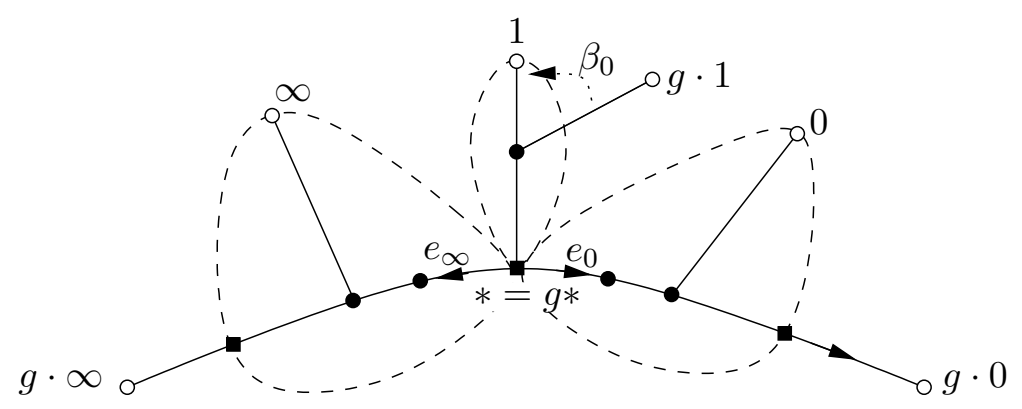

If the projection of a point at infinity to the connected union of two edges is their middle vertex, then the projection of a point at infinity to any geodesic line containing these two edges is again this middle vertex. Note that projections on closed subtrees commute with tree automorphisms. Hence the projection of $g \cdot 1$ on the geodesic line $\ell_{*}$ with points at infinity $\infty$ and 0 (which contains $e_{0}$ and $e_{\infty}$ ) is equal to $*$. The group $\underline{A}(\mathscr{O})$ acts simply transitively on $\mathscr{O}^{\times}$, which is the subset of $\partial_{\infty} \mathbb{T}=\widehat{K} \cup\{\infty\}$ consisting of elements whose projections on $\ell_{*}$ is $*$. Hence there exists $\beta_{0} \in \underline{A}(\mathscr{O})$ such that $g \beta_{0} \cdot 1=1$. Since multiplying $g$ on the right by $\beta_{0}$ does not change $g \cdot \infty$ and $g \cdot 0$, we hence have $g \beta_{0} \in \widetilde{C}$. This proves Assertion (3).

(4) Let us now fix $x \in C$, and let $g \in \widetilde{C}$ be the unique element in $\widetilde{C}$ such that $x=\Gamma g$.

Recall that any geodesic line which enters into the interior of a (closed) horoball and does not converge to its point at infinity has to exit this horoball after a finite time, and that the distance travelled inside the horoball is even. Since $g \cdot \infty$ and $g \cdot 0$ do not belong to $K$, there exists a unique sequence $\left(t_{k}=t_{k}(x)\right)_{k \in \mathbb{Z}}$ in $2 \mathbb{Z}$ such that $t_{0}=0, t_{k}<t_{k+1}$ for all $k \in \mathbb{Z}$ and

$$
g \ell_{*}(\mathbb{Z}) \cap(\Gamma *)=\left\{g \ell_{*}\left(t_{k}\right): k \in \mathbb{Z}\right\} .
$$


This sequence is hence the sequence of times at which the geodesic line $g \ell_{*}$ passes through $\Gamma *$ (normalized by being at time $t_{0}=0$ at $*$ ).

Let us fix $a \in A$ such that $x a \in C$, and let us prove that there exists a unique pair $(k, \alpha) \in \mathbb{Z} \times \underline{A}(\mathscr{O})$ such that $a=\alpha \mathfrak{a}_{0}^{t_{k}}$.

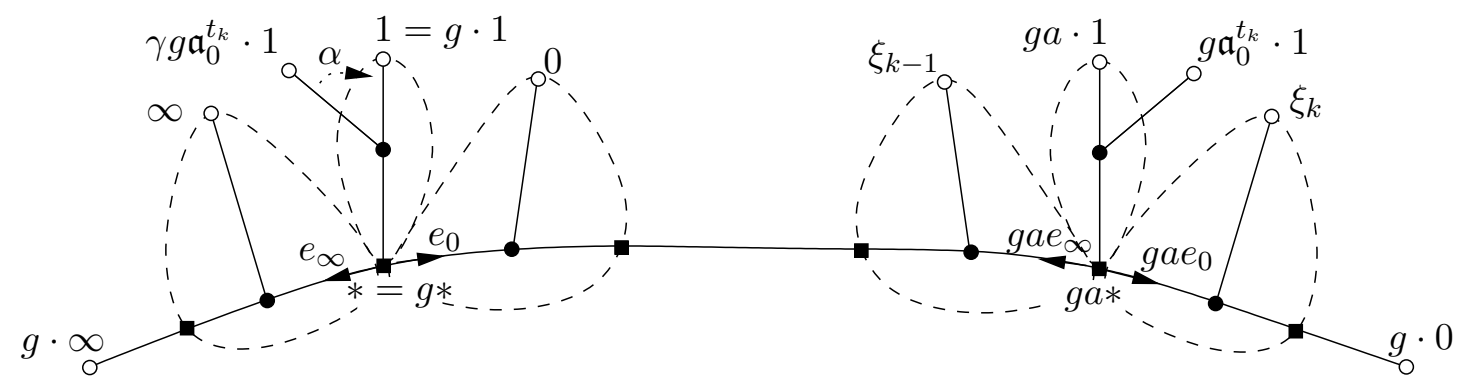

We have $g a * \in \Gamma *$ and $g a \cdot 1$ is a rational point at infinity. Indeed, since the lift of $x a$ in $\widetilde{C}$ has the form $\gamma g a$ for some $\gamma \in \Gamma$, we have $g a *=\gamma^{-1} * \in \Gamma *$ and $g a \cdot 1=\gamma^{-1} \cdot 1 \in$ $K \cup\{\infty\}$. In particular, there exists a unique $k \in \mathbb{Z}$ such that $g a *=g \mathfrak{a}_{0}^{t_{k}} *$.

Let $\xi_{k-1}$ and $\xi_{k}$ be the points at infinity of the horoballs in the family $\left(\mathscr{H}_{\xi}\right)_{\xi \in \mathbb{P}_{1}(K)}$ into which the edges $g a e_{\infty}$ and $g a e_{0}$ respectively enter.

By the simple transitivity of the projective action of $\mathrm{PGL}_{2}(K)$ on $\mathbb{P}_{1}(K)^{(3)}$, let $\gamma \in \Gamma$ be the unique element of $\Gamma$ sending $\xi_{k-1}, \xi_{k}, g a \cdot 1$ to respectively $\infty, 0,1$. Then $\gamma$ sends $g a *$ to $*, g a e_{\infty}$ to $e_{\infty}$ and $g a e_{0}$ to $e_{0}$. Therefore $\gamma g a \cdot \infty$, which is irrational since equal to $\gamma g \cdot \infty$ and $g \cdot \infty$ is irrational, is the point at infinity of a geodesic ray starting by the edge $e_{\infty}$, hence belongs to $\left({ }^{c} \mathscr{O}\right)^{\natural}$. Similarly, $\gamma g a \cdot \infty$ belongs to $\mathscr{M}^{\natural}$. As $\gamma g a \cdot 1=1$ by construction of $\gamma$, we have $\gamma g a \in \widetilde{C}$, and by Assertion (1), $\gamma$ is the unique element of $\Gamma$ such that $\gamma g a \in \widetilde{C}$.

Using arguments similar to the ones at the end of the proof of Assertion (3), since $g \mathfrak{a}_{0}^{t_{k}} \cdot 1$ projects to $g a *=g \mathfrak{a}_{0}^{t_{k}} *$ on the union of $g a e_{\infty}$ and $g a e_{0}$, the point at infinity $\gamma g \mathfrak{a}_{0}^{t_{k}} \cdot 1$ projects to $\gamma g a *=*$ on the union of $\gamma g a e_{\infty}=e_{\infty}$ and $\gamma g a e_{0}=e_{0}$. Hence there exists one and only one $\alpha \in \underline{A}(\mathscr{O})$ such that $\gamma g \mathfrak{a}_{0}^{t_{k}} \alpha \cdot 1=1$. Since $\gamma g \mathfrak{a}_{0}^{t_{k}} \alpha$ and $\gamma g a$ give the same images to $\infty, 0,1$, they are equal, hence $a=\mathfrak{a}_{0}^{t_{k}} \alpha$, for a unique $\alpha$. This proves Assertion (4).

(5) Let us fix $x \in C$. Let $n=t_{1}(x)$ be the first return time of the $A$-orbit of $x$ to $C$, and $k=\frac{n}{2}$. We denote by $g \in \widetilde{C}$ the lift of $x$ to $\widetilde{C}$. In particular, $g$ fixes $*, 1, e_{\infty}, e_{0}$.

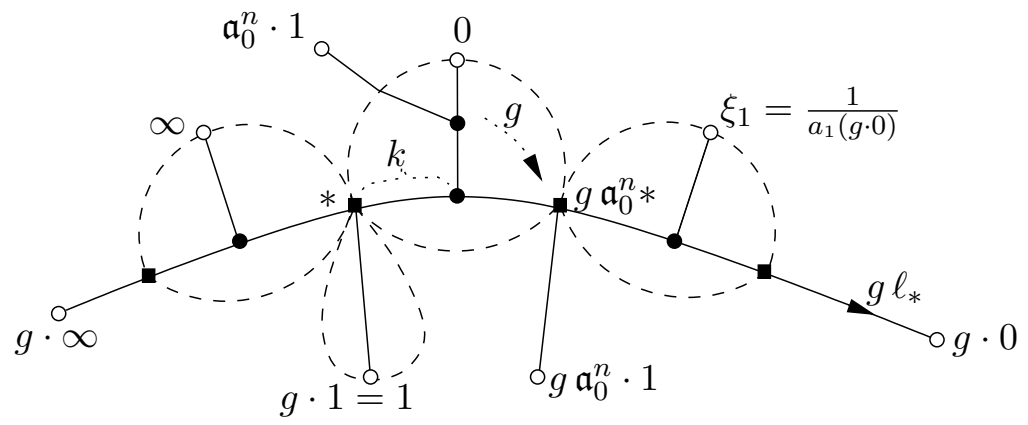

By the geometric interpretation of the continued fraction expansion of $g \cdot 0 \in \mathscr{M}$ (see [Pau, §6]), we have 
- the exiting time of $g \ell_{*}$ out of the horosphere $\mathscr{H}_{0}$ is $n$, and

$$
k=\operatorname{deg} a_{1}(g \cdot 0) ;
$$

- the point at infinity of the horoball in the family $\left(\mathscr{H}_{\xi}\right)_{\xi \in \mathbb{P}_{1}(K)}$ into which enters the geodesic line $g \ell_{*}$ after time $n$ is the first partial quotient

$$
\xi_{1}=\frac{1}{a_{1}(g \cdot 0)}
$$

of the continued fraction expansion of $g \cdot 0$.

Note that $g \mathfrak{a}_{0}^{n}$ maps $*$ to the unique intersection point of $\mathscr{H}_{0}$ and $\mathscr{H}_{\xi_{1}}$, as well as $e_{\infty}$ and $e_{0}$ to the edges starting from $g \mathfrak{a}_{0}^{n} *$ and pointing towards the points at infinity 0 and $\xi_{1}$ respectively.

Define $\gamma=\left[\begin{array}{cc}-a_{1}(g \cdot 0) & 1 \\ 1 & 0\end{array}\right]$, which belongs to $\Gamma$. Note that $\gamma$, whose associated homography is $z \mapsto \frac{1}{z}-a_{1}(g \cdot 0)$, maps 0 to $\infty$ and $\xi_{1}$ to 0 . Hence it maps the unique intersection point of $\mathscr{H}_{0}$ and $\mathscr{H}_{\xi_{1}}$, which is $g \mathfrak{a}_{0}^{n}$, to the unique intersection point of $\mathscr{H}_{\infty}$ and $\mathscr{H}_{0}$, which is $*$. It also sends the first edge of the geodesic ray from $g \mathfrak{a}_{0}^{n} *$ to 0 , which is $g \mathfrak{a}_{0}^{n} e_{\infty}$, to the first edge of the geodesic ray from $*$ to $\infty$, which is $e_{\infty}$. Similarly, $\gamma g \mathfrak{a}_{0}^{n} e_{0}=e_{0}$.

Since $\gamma g \mathfrak{a}_{0}^{n}$ fixes $e_{\infty}$ and $e_{0}$, the point at infinity $\gamma g \mathfrak{a}_{0}^{n} \cdot \infty$ (which is equal to $\gamma g \cdot \infty$ hence is irrational) belongs to ${ }^{c} \mathscr{O}$, and similarly $\gamma g \mathfrak{a}_{0}^{n} \cdot 0 \in \mathscr{M}^{\natural}$. Furthermore, as seen above, there exists a unique $\alpha \in \underline{A}(\mathscr{O})$ such that $\gamma g \mathfrak{a}_{0}^{n} \alpha \cdot 1=1$.

Therefore $\gamma g \mathfrak{a}_{0}^{n} \alpha \in \widetilde{C}$, by uniqueness $T(x)=x \mathfrak{a}_{0}^{n} \alpha$ and the unique lift of $T(x)$ in $\widetilde{C}$ is $\gamma g \mathfrak{a}_{0}^{n} \alpha$.

We now compute

$$
\begin{aligned}
\Theta \circ T(x) & =\widetilde{\Theta}\left(\gamma g \mathfrak{a}_{0}^{n} \alpha\right)=\left(\gamma g \mathfrak{a}_{0}^{n} \alpha \cdot \infty, \gamma g \mathfrak{a}_{0}^{n} \alpha \cdot 0\right)=(\gamma g \cdot \infty, \gamma g \cdot 0) \\
& =\left(\frac{1}{g \cdot \infty}-a_{1}(g \cdot 0), \frac{1}{g \cdot 0}-a_{1}(g \cdot 0)\right)=\widetilde{\Psi}(g \cdot \infty, g \cdot 0)=\widetilde{\Psi} \circ \Theta(x) .
\end{aligned}
$$

This concludes the proof of Theorem 3.

Let us introduce some notation before stating our next result. All measures in this paper are Borel and nonegative.

We denote by $\mu_{A}, \mu_{\underline{A}(\mathscr{O})}=\mu_{A \mid \underline{A}(\mathscr{O})}, \mu_{\widehat{K}}$ and $\mu_{\mathscr{M}}=\mu_{\widehat{K} \mid \mathscr{M}}$, the Haar measures of the locally compact abelian groups $A, \underline{A}(\mathscr{O}),(\widehat{K},+)$ and $(\mathscr{M},+)$ respectively, normalized as usual so that $\mu_{A}(\underline{A}(\mathscr{O}))=1$ and $\mu_{\widehat{K}}(\mathscr{O})=1$.

For every $x \in C$, we define $x_{ \pm}$by

$$
\Theta(x)=\left(x_{-}, x_{+}\right) \in\left({ }^{c} \mathscr{O}\right)^{\natural} \times \mathscr{M}^{\natural} .
$$

Let $\Theta_{2}: C \rightarrow \mathscr{M}^{\natural}$ be the map $\Theta_{2}=\operatorname{pr}_{2} \circ \Theta$, where $\mathrm{pr}_{2}:\left(\xi_{-}, \xi_{+}\right) \mapsto \xi_{+}$is the projection on the second factor, which is the continuous and surjective map on $C=\Gamma \widetilde{C}$ induced by the map $g \mapsto g \cdot 0$ from $\widetilde{C}$ to $\mathscr{M}^{\natural}$. By Theorem 3 (5), the following diagram commutes:

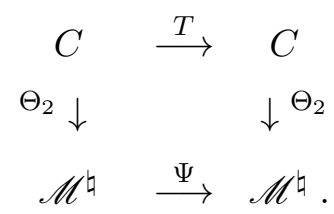


Since $\underline{A}(\mathscr{O})$ is compact and open in $G$ and by the uniqueness properties in Theorem 3 (4), the map from $C \times \underline{A}(\mathscr{O})$ to $X$ defined by $(x, a) \mapsto x a$ is a homeomorphism onto its image, which is an open neighborhood of $C$ in $X$. Denoting by $C \underline{A}(\mathscr{O})$ its image, the map $C \underline{A}(\mathscr{O}) \rightarrow C$ defined by $x a \mapsto x$ for all $x \in C$ and $a \in \underline{A}(\mathscr{O})$ is a fibration with fibers the right orbits of $\underline{A}(\mathscr{O})$. The product group $T^{\mathbb{Z}} \times \underline{A}(\mathscr{O})$ acts in the obvious way on $C \underline{A}(\mathscr{O})$. We still denote by $t_{k}: C \underline{A}(\mathscr{O}) \rightarrow \mathbb{Z}$ the $k$-th return time in $C \underline{A}(\mathscr{O})$ of the orbits under $\mathfrak{a}_{0}^{\mathbb{Z}}$, that is $t_{k}(x a)=t_{k}(x)$ for all $x \in C$ and $a \in \underline{A}(\mathscr{O})$. Given any measure $\mu$ on $C$, we denote by $\mu \otimes \mu_{\underline{A}(\mathscr{O})}$ the pushforwards by $(x, a) \mapsto x a$ of the product measure of $\mu$ and $\mu_{\underline{A}(\mathscr{O})}$, which is a finite nonzero measure on $C \underline{A}(\mathscr{O})$ if $\mu$ is finite and nonzero.

We denote by $M_{A}$ the space of finite nonzero $A$-invariant measures on $X^{\natural}$, by $M_{T^{\mathbb{Z}}} \underline{A(\mathscr{O})}$ the space of finite nonzero $T^{\mathbb{Z}} \times \underline{A}(\mathscr{O})$-invariant measures $m$ on $C \underline{A}(\mathscr{O})$ such that the integral $\int_{C \underline{A}(\mathscr{O})} t_{1} d m$ is finite and nonzero, by $M_{T}$ the space of $T$-invariant measures $m$ on $C$ such that $\int_{C} t_{1} d m$ is finite and nonzero, and by $M_{\mathscr{M}}$ the space of $\Psi$-invariant measures $m$ on $\mathscr{M}$ such that $\int_{f \in \mathscr{M}} \operatorname{deg}\left(a_{1}(f)\right) d m(f)$ is finite and nonzero. We endow these spaces with their weak-star topologies.

We have maps

$$
M_{A} \stackrel{F_{1}}{\longrightarrow} M_{T^{\mathbb{Z}} \underline{A}(\mathscr{O})} \stackrel{F_{2}}{\longleftarrow} M_{T} \stackrel{\left(\Theta_{2}\right)_{*}}{\longrightarrow} M_{\mathscr{M}},
$$

where $F_{1}: m \mapsto m_{\mid C \underline{A}(\mathscr{O})}, F_{2}: \mu \mapsto \mu \otimes \mu_{\underline{A}(\mathscr{O})}$, and we define (see the proof below for the invertibility of $F_{2}$ )

$$
F=\left(\Theta_{2}\right)_{*} \circ\left(F_{2}\right)^{-1} \circ F_{1}: M_{A} \rightarrow M_{\mathscr{M}} .
$$

We denote by $\delta_{x}$ the unit Dirac mass at any point $x$ in any measurable space.

The following result strengthens the statement announced in the Introduction relating $A$-orbits to $\Psi$-orbits, and gives the measure-theoretic correspondence. The use of semigroups comes from the fact that $\Psi$ is non invertible. Recall that the analogue, for the Artin map $\Psi: x \mapsto \frac{1}{x}-\left[\frac{1}{x}\right]$, of the Gauss measure on [0,1] for the Gauss map $x \mapsto \frac{1}{x}-\left\lfloor\frac{1}{x}\right\rfloor$, is the Haar probability measure of the additive group $\mathscr{M}$ (see for instance [BP, Prop. 5.6] for a dynamical explanation).

Theorem 4 (1) For every $x \in C$, the image by $\Theta_{2}$ of the intersection with $C$ of the orbit of $x$ by right translations under the semigroup $A_{+}=\underline{A}(\mathscr{O}) \mathfrak{a}_{0}^{\mathbb{N}}$ is equal to the $\Psi$-orbit of $\Theta_{2}(x)$ :

$$
\Theta_{2}\left(C \cap\left(x A_{+}\right)\right)=\Psi^{\mathbb{N}}\left(\Theta_{2}(x)\right) .
$$

In particular, if $x \in C$ is A-periodic, then $\Theta_{2}(x)$ is a quadratic irrational. Furthermore, the period of $x \in C$ under the iteration of $T$ is equal to the period of the periodic part of the continued fraction expansion of $\Theta_{2}(x)$.

(2) The map $F: M_{A} \rightarrow M_{\mathscr{M}}$ is a homeomorphism for the weak-star topologies, such that $F\left(c m+m^{\prime}\right)=c F(m)+F\left(m^{\prime}\right)$ and $F(m) \leq F\left(m^{\prime \prime}\right)$ for all $c>0$ and $m, m^{\prime}, m^{\prime \prime} \in M_{A}$ such that $m \leq m^{\prime \prime}$.

(3) The restriction to $C \underline{A}(\mathscr{O})$ of the $G$-invariant probability measure $m_{X}$ on $X$ disintegrates by the fibration $C \underline{A}(\mathscr{O}) \rightarrow C$, with conditional measures the $\underline{A}(\mathscr{O})$-invariant probability measures on the fibers, over a finite measure $m_{C}$ on $C$ such that, for every $x \in C$, using the homeomorphism $\Theta: x \mapsto\left(x_{-}, x_{+}\right)$,

$$
d m_{C}(x)=\frac{q(q-1)^{2}}{2} \frac{d \mu_{\widehat{K}}\left(x_{-}\right)}{\left|x_{-}\right|_{\infty}^{4}} d \mu_{\widehat{K}}\left(x_{+}\right) .
$$


Furthermore, the pushforwards under $\Theta_{2}$ of $m_{C}$ is equal to a multiple of the Haar measure on $\mathscr{M}$ :

$$
\left(\Theta_{2}\right)_{*} m_{C}=\frac{q^{4}(q-1)^{2}}{2\left(q^{2}+q+1\right)} \mu_{\mathscr{M}},
$$

and the measure on $\mathscr{M}$ corresponding to the $G$-invariant probability measure $m_{X}$ on $X$ is a multiple of the Haar measure on $\mathscr{M}$ :

$$
F\left(m_{X}\right)=\frac{q^{4}(q-1)^{2}}{2\left(q^{2}+q+1\right)} \mu_{\mathscr{M}} .
$$

(4) If $x \in C$ is A-periodic in $X$, then for $k$ big enough, the measure on $\mathscr{M}$ corresponding to the A-invariant probability measure $\mu_{x}$ on the $A$-orbit of $x$, normalized to be a probability measure, is equal to the equiprobability on the (finite) $\Psi$-orbit of $\Theta_{2}\left(T^{k} x\right)$ : More precisely, if $n_{x}$ is the period of the periodic part of the continued fraction expansion of $\Theta_{2}(x)$, then

$$
F\left(\mu_{x}\right)=\frac{1}{2 \sum_{n=k}^{k+n_{x}-1} \operatorname{deg} a_{n}\left(\Theta_{2}(x)\right)} \sum_{0 \leq n \leq n_{x}-1} \delta_{\Psi^{n+k}\left(\Theta_{2}(x)\right)} .
$$

In the last claim, we may take $k=0$ if $x$ is $A_{+}$-periodic, and in general $k$ may be taken to be any time after which the continued fraction expansion $\left(a_{n}\left(\Theta_{2}(x)\right)\right)_{n \geq 1}$ of $\Theta_{2}(x)$ is periodic.

Proof. (1) It follows from Theorem 3 (4) that

$$
C \cap\left(x A_{+}\right)=\left\{x \alpha_{k} \mathfrak{a}_{0}{ }^{t_{k}}: k \in \mathbb{N}\right\}=\left\{T^{k}(x): k \in \mathbb{N}\right\} .
$$

The result then follows from the commutativity of the diagram in Equation (3).

(2) Step 1 : It is clear that the map $\left(\Theta_{2}\right)_{*}: M_{T} \rightarrow M_{\mathscr{M}}$ is order preserving and linear (for positive scalars). Let us prove that it is a weak-star homeomorphism. There is a direct proof of this, but we will use a symbolic dynamics argument, as it is illuminating.

For every $x \in C$, let $\left[0 ; a_{1}, a_{2}, \ldots\right]$ be the continued fraction expansion of $x_{+} \in \mathscr{M}^{\natural}$ and let $\left[a_{0} ; a_{-1}, a_{-2}, \ldots\right]$ be the continued fraction expansion of $x_{-} \in\left({ }^{c} \mathscr{O}\right)^{\natural}$, so that $a_{i} \in$ $R-\mathbb{F}_{q}$ for all $i \in \mathbb{Z}$. Let $\sigma:\left(R-\mathbb{F}_{q}\right)^{\mathbb{Z}} \rightarrow\left(R-\mathbb{F}_{q}\right)^{\mathbb{Z}}$ be the (two-sided) shift defined by $\left(\sigma\left(\left(x_{i}\right)_{i \in \mathbb{Z}}\right)\right)_{i}=x_{i+1}$ for all $i \in \mathbb{Z}$. Then by [BP, Theo. 3.7] and Theorem $3(2)$ and (5), the map $\Xi: C \rightarrow\left(R-\mathbb{F}_{q}\right)^{\mathbb{Z}}$ defined by $x \mapsto\left(a_{i}\right)_{i \in \mathbb{Z}}$ is a homeomorphism such that the following diagram commutes

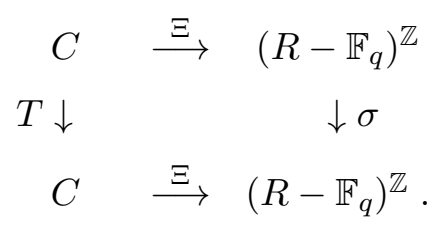

Let $\sigma_{+}:\left(R-\mathbb{F}_{q}\right)^{\mathbb{N}-\{0\}} \rightarrow\left(R-\mathbb{F}_{q}\right)^{\mathbb{N}-\{0\}}$ be the (one-sided) shift defined by the formula $\left(\sigma_{+}\left(\left(x_{i}\right)_{i \in \mathbb{N}-\{0\}}\right)\right)_{i}=x_{i+1}$ for all $i \in \mathbb{N}-\{0\}$. By the properties of the continued fraction expansions, the map cfe : $\mathscr{M}^{\natural} \rightarrow\left(R-\mathbb{F}_{q}\right)^{\mathbb{N}-\{0\}}$ defined by $f \mapsto\left(a_{i}(f)\right)_{i \in \mathbb{N}-\{0\}}$ is a 
homeomorphism such that the following diagram commutes

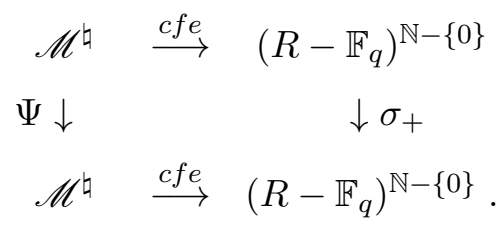

Let $\pi_{+}:\left(R-\mathbb{F}_{q}\right)^{\mathbb{Z}} \rightarrow\left(R-\mathbb{F}_{q}\right)^{\mathbb{N}-\{0\}}$ be the map, called the natural extension, forgetting the past, defined by $\pi_{+}\left(\left(x_{i}\right)_{i \in \mathbb{Z}}\right)=\left(x_{i}\right)_{i \in \mathbb{N}-\{0\}}$. The following diagram commutes by construction

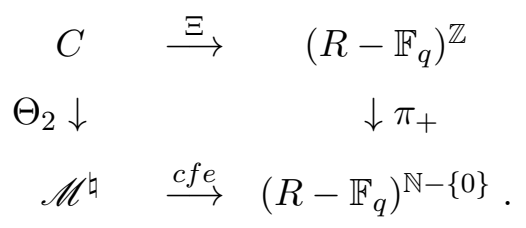

Note that, as already seen, for every $x \in C$,

$$
t_{1}(x)=2 \operatorname{deg} a_{1}\left(\Theta_{2}(x)\right),
$$

so that $\int_{\mathscr{M}} \operatorname{deg} a_{1} d\left(\Theta_{2}\right)_{*} \mu=\frac{1}{2} \int_{C} t_{1} d \mu$ for every measure $\mu$ on $C$.

Step 1 now follows, by conjugation of dynamical systems, from the well-known fact (see for instance [Kit], or use the fact that the symbolic Borel $\sigma$-algebras are generated by the cylinders and Kolmogorov's extension theorem) that the pushforwards map $\left(\pi_{+}\right)_{*}$ is a weak-star homeomorphism between the space of $\sigma$-invariant measures on $\left(R-\mathbb{F}_{q}\right)^{\mathbb{Z}}$ for which $\operatorname{deg} a_{1}$ is integrable and the space of $\sigma_{+}$-invariant measures on $\left(R-\mathbb{F}_{q}\right)^{\mathbb{N}-\{0\}}$ for which $\operatorname{deg} a_{1}$ is integrable.

Step 2 : Let us prove that the map $F_{2}: M_{T} \rightarrow M_{T^{\mathbb{Z}} \underline{A}(\mathscr{O})}$ is a weak-star homeomorphism. Since $\int_{C \underline{A}(\mathscr{O})} t_{1} d\left(\mu \otimes \mu_{\underline{A}(\mathscr{O})}\right)=\int_{C} t_{1} d \mu$ for every measure $\mu$ on $C$, and since $T^{\mathbb{Z}}$ and $\underline{A}(\mathscr{O})$ commute, the measure $\mu \otimes \mu_{\underline{A}(\mathscr{O})}$ is indeed $T^{\mathbb{Z}} \times \underline{A}(\mathscr{O})$-invariant if $\mu$ is $T$ invariant, hence $F_{2}$ is well defined. It is clear that $F_{2}$ is order preserving and linear.

Every finite nonzero $T^{\mathbb{Z}} \times \underline{A}(\mathscr{O})$-invariant measure $m$ on $C \underline{A}(\mathscr{O})$ disintegrates by the fibration $C \underline{A}(\mathscr{O}) \rightarrow C$. The conditional measures on the fibers, which are $\underline{A}(\mathscr{O})$-invariant and may be assumed to be probability measures, are hence equal to the $\underline{A}(\mathscr{O})$-invariant probability measures, by uniqueness. Since the return time $t_{1}$ is constant on the fibers, the measure on $C$ over which $m$ disintegrates hence belongs to $M_{T}$ if $m$ belongs to $M_{T^{\mathbb{Z}} \underline{A(\mathscr{O})} \text { ) }}$. Step 2 follows.

Step 3 : Let us finally prove that the map $F_{1}: M_{A} \rightarrow M_{T^{\mathbb{Z}} A(\mathscr{O})}$ is a weak-star homeomorphism. Since it is clear that $F_{1}$ is order preserving and linear, Steps 1 to 3 prove Assertion (2) of Theorem 4 by composition.

Since $C \underline{A}(\mathscr{O})$ is open in $X^{\natural}$, and since any $A$-orbit in $X^{\natural}$ meets $C$, for every finite nonzero $A$-invariant measure $m$ on $X^{\natural}$, the measure $m_{\mid C \underline{A}(\mathscr{O})}$ is finite and nonzero. The open subset $C \underline{A}(\mathscr{O})$ is a transversal for the action of $\mathfrak{a}_{0}$. If $t_{0}: X^{\natural} \rightarrow \mathbb{N}$ is the first passage time in $C \underline{A}(\mathscr{O})$ of the orbit of $\mathfrak{a}_{0}$, that is,

$$
t_{0}: x \mapsto \min \left\{n \in \mathbb{N}: \mathfrak{a}_{0}^{n} x \in C \underline{A}(\mathscr{O})\right\},{ }^{4}
$$

\footnotetext{
${ }^{4}$ This map from $X^{\natural}$ to $\mathbb{N}$ extends to $X^{\natural}$ the map from $C \underline{A}(\mathscr{O})$ to $\mathbb{N}$ previously defined in Theorem $3(4)$ to be the zero map.
} 
and if $T_{0}: X^{\natural} \rightarrow C \underline{A}(\mathscr{O})$ is the first passage map $x \mapsto \mathfrak{a}_{0}^{t_{0}(x)} x$ in $C \underline{A}(\mathscr{O})$ of the orbit of $\mathfrak{a}_{0}$, then $\left\{t \in \mathbb{Z}: \mathfrak{a}_{0}^{t} x \in C \underline{A}(\mathscr{O})\right\}$ is exactly the set $\left\{t_{0}(x)+t_{k}\left(T_{0}(x)\right): k \in \mathbb{Z}\right\}$. Since $C \underline{A}(\mathscr{O})$ is open, the disintegration of any $A$-invariant measure with respect to this transversal is simply the restriction of $m$ to $C \underline{A}(\mathscr{O})$, and this restriction is $\underline{A}(\mathscr{O})$-invariant. Therefore the discrete time dynamical system $\left(X^{\natural}, \mathfrak{a}_{0}\right)$ identifies with the discrete time suspension of the discrete time dynamical system $(C \underline{A}(\mathscr{O}), T)$ with roof function $t_{1}$. Furthermore, any measure on $C \underline{A}(\mathscr{O})$ which is invariant both under $T$ and $\underline{A}(\mathscr{O})$ suspends to an $A$-invariant measure, whose total mass is the integral of the roof function. Step 3 follows.

(3) The proof of the first claim in Assertion (3) of Theorem 4, that is, the computation of

$$
m_{C}=\left(F_{2}\right)^{-1} \circ F_{1}\left(m_{X}\right),
$$

follows from the next proposition.

Proposition 5 The restriction $F_{1}\left(m_{X}\right)$ to $C \underline{A}(\mathscr{O})$ of the $G$-invariant probability measure $m_{X}$ on $X$ satisfies, for all $x \in C$ and $a \in \underline{A}(\mathscr{O})$, using the homeomorphism $x a \mapsto$ $\left(x_{-}, x_{+}, a\right)$ from $C \underline{A}(\mathscr{O})$ to $\left({ }^{c} \mathscr{O}\right)^{\natural} \times \mathscr{M}^{\natural} \times \underline{A}(\mathscr{O})$,

$$
d m_{X}(x a)=\frac{q(q-1)^{2}}{2} \frac{d \mu_{\widehat{K}}\left(x_{-}\right)}{\left|x_{-}\right|_{\infty}^{4}} d \mu_{\widehat{K}}\left(x_{+}\right) d \mu_{A}(a) .
$$

Proof. Let us denote by $d v$ the counting measure on the discrete set $V \mathbb{T}$. Recall that Hopf's parametrisation is the homeomorphism from $\mathscr{G} \mathbb{T}$ onto its image in $\partial_{\infty} \mathbb{T} \times \partial_{\infty} \mathbb{T} \times V \mathbb{T}$ defined by $\ell \mapsto(\ell(-\infty), \ell(+\infty), \ell(0))$. Let $\widetilde{m}_{\mathrm{BM}}$ be the (locally finite, positive, Borel) measure on $\mathscr{G} \mathbb{T}$ defined, using Hopf's parametrisation, on the full measure set of $\ell \in \mathscr{G} \mathbb{T}$ such that $\ell( \pm \infty) \neq \infty$, by

$$
d \widetilde{m}_{\mathrm{BM}}(\ell)=\frac{d \mu_{\mathrm{Hau}}(\ell(-\infty)) d \mu_{\mathrm{Hau}}(\ell(-\infty)) d v(\ell(0))}{|\ell(-\infty)-\ell(+\infty)|_{\infty}^{2}}
$$

By well-known arguments of for instance [BuM], [Rob], [BP, §4] and [BPP, §4.4], since $\Gamma$ is a lattice in $G$, the measure $\widetilde{m}_{\mathrm{BM}}$ is invariant on the left by the action of $\Gamma$ and on the right by the geodesic flow, and defines a finite nonzero measure $m_{\mathrm{BM}}$ on $\Gamma \backslash \mathscr{G} \mathbb{T}$ invariant by the geodesic flow (called the Bowen-Margulis measure). Furthermore, the $G$-invariant probability measure $m_{X}$ on $X$ disintegrates with respect to the fiber bundle $X=\Gamma \backslash G \rightarrow \Gamma \backslash \mathscr{G} \mathbb{T}=\Gamma \backslash G / \underline{A}(\mathscr{O})$ defined by $\Gamma g \mapsto \Gamma g \ell_{*}$, over the probability measure $\frac{m_{\mathrm{BM}}}{\left\|m_{\mathrm{BM}}\right\|}$, with conditionnal measures the $\underline{A}(\mathscr{O})$-invariant probability measures on the fibers.

Recall that for all $x \in C$, we have $x_{-}=g \cdot \infty=g \ell_{*}(-\infty)$ and $x_{+}=g \cdot 0=g \ell_{*}(+\infty)$ where $g$ is the unique lift of $x$ in $\widetilde{C}$; furthermore $g \ell_{*}(0)=g *=*$ is constant. As seen during the proof of Theorem 3, if $\gamma \in \Gamma$ fixes $g \ell_{*}$ for some $g \in \widetilde{C}$, then it fixes $*, e_{\infty}$ and $e_{0}$, hence it belongs to $\mathrm{PGL}_{2}(\mathscr{O}) \cap \Gamma=\mathrm{PGL}_{2}\left(\mathbb{F}_{q}\right)$ and is diagonal. But a nontrivial element of $\mathrm{PGL}_{2}\left(\mathbb{F}_{q}\right) \cap A$ does not fix an irrational point at infinity. Hence the stabilizer in $\Gamma$ of an element of $\widetilde{C} \ell_{*}$ is trivial. By Equation (4), we thus have, for all $x \in C$ and $a \in \underline{A}(\mathscr{O})$,

$$
d m_{X}(x a)=\frac{1}{\left\|m_{\mathrm{BM}}\right\|} \frac{d \mu_{\mathrm{Hau}}\left(x_{-}\right) d \mu_{\mathrm{Hau}}\left(x_{+}\right)}{\left|x_{-}-x_{+}\right|_{\infty}^{2}} d \mu_{A}(a) .
$$

For all $x \in C$, we have $x_{-} \in{ }^{c} \mathscr{O}$ and $x_{+} \in \mathscr{M}$, hence $\left|x_{+}\right|_{\infty}<1<\left|x_{-}\right|_{\infty}$, so that $\left|x_{-}-x_{+}\right|_{\infty}=\left|x_{-}\right|_{\infty}$. By [BPP, Prop. 15.2(1)] (where $\mu_{\text {Hau }}$ was normalized in this 
reference to have total mass $\frac{q+1}{q}$ instead of 1 , by [loc. cit., Prop. 15.2 (2)]), on the set of $\xi \in \partial_{\infty} \mathbb{T}=\widehat{K} \cup\{\infty\}$ such that $\xi \neq \infty$, we have

$$
d \mu_{\mathrm{Hau}}(\xi)=\frac{q}{q+1} \frac{d \mu_{\widehat{K}}(\xi)}{\max \left\{1,|\xi|_{\infty}^{2}\right\}} .
$$

Note that $\left\|m_{\mathrm{BM}}\right\|=\frac{2}{q(q-1)^{2}}\left(\frac{q}{q+1}\right)^{2}$, by [BPP, Prop. 15.3 (1)] (again using the above difference of normalization). Hence

$$
d m_{X}(x a)=\frac{q(q-1)^{2}}{2} \frac{d \mu_{\widehat{K}}\left(x_{-}\right)}{\left|x_{-}\right|_{\infty}^{4}} d \mu_{\widehat{K}}\left(x_{+}\right) d \mu_{A}(a) .
$$

The result follows.

By the first claim in Assertion (3) of Theorem 4, we immediately have that there exists a constant $c>0$ such that $\left(\Theta_{2}\right)_{*} m_{C}=c \mu_{\widehat{K} \mid \mathscr{M}^{\natural}}=c \mu_{\mathscr{M}}$. We have $\mu_{\widehat{K}}(\mathscr{M})=\frac{1}{q}$ by the normalization of $\mu_{\widehat{K}}$ and the fact that $\mathscr{O}=\sqcup_{x \in \mathbb{F}_{q}}(x+\mathscr{M})$. This implies that $c=q\left\|m_{C}\right\|=q m_{X}(C \underline{A}(\mathscr{O}))$.

Note that $\widehat{K}-\mathscr{O}=\sqcup_{u \in R-\mathbb{F}_{q}}(u+\mathscr{M})$, and that there are $(q-1) q^{n}$ polynomials in $R$ with degree $n$ for every $n \in \mathbb{N}$. Hence, by Proposition 5 , we have

$$
\begin{aligned}
m_{X}(C \underline{A}(\mathscr{O})) & =\frac{q(q-1)^{2}}{2} \int_{c \mathscr{O}} \frac{d \mu_{\widehat{K}}\left(x_{-}\right)}{\left|x_{-}\right|_{\infty}^{4}} \mu_{\widehat{K}}(\mathscr{M}) \mu_{A}(\underline{A}(\mathscr{O})) \\
& =\frac{(q-1)^{2}}{2} \sum_{u \in R-\mathbb{F}_{q}} \int_{y \in \mathscr{M}} \frac{d \mu_{\widehat{K}}(y)}{|u+y|_{\infty}^{4}}=\frac{(q-1)^{2}}{2} \sum_{u \in R-\mathbb{F}_{q}} \frac{\mu_{\widehat{K}}(\mathscr{M})}{|u|_{\infty}^{4}} \\
& =\frac{(q-1)^{2}}{2 q} \sum_{n=1}^{+\infty} \frac{(q-1) q^{n}}{q^{4 n}}=\frac{q^{3}(q-1)^{2}}{2\left(q^{2}+q+1\right)} .
\end{aligned}
$$

This proves the second claim in Assertion (3) of Theorem 4, and the last one follows by composition.

(4) Let us fix $x \in C$ which is $A$-periodic in $X^{\natural}$. As seen in Assertion (1), $x$ is periodic under $T$. Up to replacing $x$ by $T^{k} x$ for any $k$ big enough, since $T^{k} x \in x A$ so that $\mu_{T^{k} x}=\mu_{x}$, we may assume by Equation (3) that the continued fraction expansion of $\Theta_{2}(x)$ is periodic. We then have

$$
C \underline{A}(\mathscr{O}) \cap x A=\left\{T^{n}(x) \underline{A}(\mathscr{O}): 0 \leq n<n_{x}\right\} .
$$

Furthermore, for all $y \in C$ and $a \in \underline{A}(\mathscr{O})$,

$$
d \mu_{x \mid C \underline{A}(\mathscr{O})}(y a)=\frac{\mu_{x}(C \underline{A}(\mathscr{O}))}{n_{x}} \sum_{n=0}^{n_{x}-1} \delta_{T^{n}(x)}(y) d \mu_{A}(a),
$$

where $\mu_{x}(C \underline{A}(\mathscr{O}))=\frac{n_{x}}{\sum_{n=0}^{n_{x}-1} t_{1}\left(T^{n}(x)\right)}$ is the proportion of points of the $\mathfrak{a}_{0}^{\mathbb{Z}}$-orbit of $x$ that are in $C \underline{A}(\mathscr{O})$. Recalling that the first return time satisfies $t_{1}(x)=2 \operatorname{deg} a_{1}\left(\Theta_{2}(x)\right)$ and by the commutativity of the diagram in Equation (3), this proves the result. 


\section{Behavior of continued fraction expansions along Hecke rays}

In this section, we give more precisions on the correspondence between $A$-orbits in the moduli space $X$ and $\Psi$-orbits in $\mathscr{M}$, for the case of quadratic irrationals. We study the behavior of the degrees of the coefficients of the continued fraction expansions of the images in $\mathscr{M}$ of $A$-periodic elements in $X$ varying along Hecke rays, and we prove Theorem 1 and Theorem 2.

We start by recalling some definitions from for instance [KePS]. For every $x \in X$ and every prime polynomial $P$ in $R$, the $P$-Hecke tree $T_{P}(x)$ with root $x$ is the connected component of $x$ in the graph with vertex set $X$, with an edge between the homothety classes of two $R$-lattices $\Lambda$ and $\Lambda^{\prime}$ when $\Lambda^{\prime} \subset \Lambda$ and $\Lambda / \Lambda^{\prime}$ is isomorphic to $R / P R$ as an $R$-module. If $x=\Gamma g=g^{-1}[R \times R]$, we hence have

$$
T_{P}(x)=\left\{g^{-1} \gamma\left[R \times P^{n} R\right]: \gamma \in \Gamma, n \in \mathbb{N}\right\} .
$$

The boundary at infinity of $T_{P}(x)$ identifies with the projective line $\mathbb{P}_{1}\left(K_{P}\right)$ over the completion $K_{P}$ of $K$ for the $P$-adic absolute value $|x|_{P}=q^{-v_{P}(x)}$, where $v_{P}\left(P^{m} \frac{A}{B}\right)=m$ for all $m \in \mathbb{Z}$ and $A, B \in R$ not divisible by $P$ (with $B \neq 0$ ). A geodesic ray starting from $x$ (called a Hecke ray) in $T_{P}(x)$ is called rational if its point at infinity belongs to $\mathbb{P}_{1}(K)$, that is, if it is of the form $n \mapsto g^{-1} \gamma\left[R \times P^{n} R\right]$ for some fixed $\gamma \in \Gamma$. We refer for instance to $[\mathrm{KePS}, \S 1]$ for details and more informations.

Now let us fix $f \in Q I$. We denote by $f^{\sigma} \in Q I$ the Galois conjugate of $f$ over $K$. Let $g_{f}=\left[\begin{array}{cc}f^{\sigma} & f \\ 1 & 1\end{array}\right] \in \mathrm{PGL}_{2}(\widehat{K})$. Let $t_{f}=\frac{f^{\sigma}-1}{1-f} \in \widehat{K}$ and $a_{f}=\alpha_{0}\left(t_{f}\right)=\left[\begin{array}{cc}1 & 0 \\ 0 & t_{f}\end{array}\right] \in A$. Finally, let

$$
x_{f}=\Gamma g_{f} a_{f} \in X
$$

Note that for all $\left(\begin{array}{ll}a & b \\ c & d\end{array}\right) \in \mathrm{GL}_{2}(K)$, we have

$$
\left(\begin{array}{ll}
a & b \\
c & d
\end{array}\right)\left(\begin{array}{cc}
f^{\sigma} & f \\
1 & 1
\end{array}\right)=\left(\begin{array}{cc}
\frac{a f^{\sigma}+b}{c f^{\sigma}+d} & \frac{a f+b}{c f+d} \\
1 & 1
\end{array}\right)\left(\begin{array}{cc}
c f^{\sigma}+d & 0 \\
0 & c f+d
\end{array}\right) .
$$

Hence for every $\gamma \in \mathrm{PGL}_{2}(K)$, we have

$$
x_{f} A=\Gamma \gamma g_{f} a_{f} A=\Gamma g_{\gamma \cdot f} A=x_{\gamma \cdot f} A .
$$

Lemma 6 The points $\Gamma g_{f}$ and $x_{f}$ of $X$ are $A$-periodic. If $f \in \mathscr{M}$ and $f^{\sigma} \in{ }^{c} \mathscr{O}$, then $a_{f} \in A_{+}, x_{f} \in C$ and $\Theta\left(x_{f}\right)=\left(f^{\sigma}, f\right)$, so that $\Theta_{2}\left(x_{f}\right)=f$.

Proof. Let $\gamma \in \Gamma-\{$ id $\}$ projectively fixing $f$ (hence $f^{\sigma}$ ), with $v_{\infty}(\operatorname{tr} \widetilde{\gamma}) \neq 0$ for some lift $\widetilde{\gamma} \in \mathrm{GL}_{2}(R)$ of $\gamma$. The existence of $\gamma$ is classical, see for instance [Ser, KePS] and Proposition 17.2 with Equation (15.6) in [BPP]. By Equation (5), there exists $w \in R+f R$

such that $\gamma g_{f}=g_{f}\left[\begin{array}{cc}w^{\sigma} & 0 \\ 0 & w\end{array}\right]=g_{f} \alpha_{0}(t)$, with $v_{\infty}(t)=v_{\infty}(\operatorname{tr} \widetilde{\gamma}) \neq 0$. The fact that $\Gamma g_{f}$ (and hence $x_{f}$, which is in the same $A$-orbit) is $A$-periodic then follows for instance from [KePS, Prop. 11].

Now assume that $f \in \mathscr{M}$ and $f^{\sigma} \in{ }^{c} \mathscr{O}$. Then $v_{\infty}\left(t_{f}\right)=v_{\infty}\left(f^{\sigma}\right)<0$, hence $a_{f} \in A_{+}$. 
Let $g_{f}^{\prime}=g_{f} a_{f}$. An easy computation gives

$$
g_{f}^{\prime} \cdot(\infty, 0,1)=\left[\begin{array}{cc}
f^{\sigma} & f \\
1 & 1
\end{array}\right]\left[\begin{array}{cc}
1 & 0 \\
0 & t_{f}
\end{array}\right] \cdot(\infty, 0,1)=\left(f^{\sigma}, f, 1\right) .
$$

Hence $g_{f}^{\prime} \in \widetilde{C}$, thus $x_{f}=\Gamma g_{f}^{\prime} \in C=\Gamma \widetilde{C}$. We have

$$
\Theta\left(x_{f}\right)=\widetilde{\Theta}\left(g_{f}^{\prime}\right)=\left(g_{f}^{\prime} \cdot \infty, g_{f}^{\prime} \cdot 0\right)=\left(g_{f} \cdot \infty, g_{f} \cdot 0\right)=\left(f^{\sigma}, f\right),
$$

so that $\Theta_{2}\left(x_{f}\right)=\operatorname{pr}_{2} \circ \Theta\left(x_{f}\right)=f$.

Proof of Theorem 1. Let $f \in Q I$.

Let us prove the first assertion of Theorem 1. We fix an irreducible polynomial $P \in R$. For every $n \in \mathbb{N}$, let $\gamma_{n} \in \Gamma$ be such that the quadratic irrational

$$
f_{n}=\gamma_{n} \cdot\left(P^{n} f\right)
$$

satisfies $f_{n} \in \mathscr{M}$ and $f_{n}{ }^{\sigma} \in{ }^{c} \mathscr{O}$. Since the projective action of $\Gamma=\operatorname{PGL}_{2}(R)$ on $Q I$ does not change the period of the eventually periodic continued fraction expansion of $f$, up to cyclic permutation and multiplications by elements of $\mathbb{F}_{q}^{\times}$(which do not change the degrees), there exist $k_{n}, k_{n}^{\prime} \in \mathbb{N}-\{0\}$ such that the continued fraction expansions of $f_{n}$ and $P^{n} f$ are indeed periodic after the times $k_{n}$ and $k_{n}^{\prime}$ respectively, with the same period length $\ell_{n} \in \mathbb{N}-\{0\}$, such that for every $i \in \mathbb{N}$,

$$
\operatorname{deg} a_{k_{n}+i}\left(P^{n} f\right)=\operatorname{deg} a_{k_{n}^{\prime}+i}\left(f_{n}\right) .
$$

By Equation (5), for every $n \in \mathbb{N}$, there exists $b_{n} \in A$ such that $g_{f_{n}} b_{n}=\gamma_{n} g_{P^{n} f}$. Hence

$$
\begin{aligned}
\Gamma g_{f_{n}} b_{n} & =\left(g_{f_{n}} b_{n}\right)^{-1}[R \times R]=\left(\gamma_{n} g_{P^{n} f}\right)^{-1}[R \times R]=g_{f}^{-1}\left[\begin{array}{cc}
P^{n} & 0 \\
0 & 1
\end{array}\right]^{-1} \gamma_{n}^{-1}[R \times R] \\
& =g_{f}^{-1}\left[R \times P^{n} R\right] .
\end{aligned}
$$

Therefore $\left(\Gamma g_{f_{n}} b_{n}\right)_{n \in \mathbb{N}}$ is the sequence of vertices along a rational Hecke ray in the $P$-Hecke tree $T_{P}\left(\Gamma g_{f}\right)$. Note that $\Gamma g_{f}$ is $A$-periodic by the first claim of Lemma 6 .

For every $n \in \mathbb{N}$, the point $x_{f_{n}}=\Gamma g_{f_{n}} a_{f_{n}}$, which belongs to $C$ by Lemma 6 , is in the same $A$-orbit as $\Gamma g_{f_{n}} b_{n}$. Let $\lambda_{n} \in \mathbb{N}-\{0\}$ be the period of $\Gamma g_{f_{n}} a_{f_{n}} \ell_{*}$ under the geodesic flow on $\Gamma \backslash \mathscr{G} \mathbb{T}$, which is also the period of $\Gamma g_{f_{n}} b_{n} \ell_{*}$, since $A$ preserves $\ell_{*}$.

Since $\ell_{*}(+\infty)=0$, the geodesic line $g_{f_{n}} a_{f_{n}} \ell_{*}$, through $*$ at time $t=0$, has point at $+\infty$ equal, using Lemma 6 , to

$$
g_{f_{n}} a_{f_{n}} \cdot 0=\Theta_{2}\left(x_{f_{n}}\right)=f_{n} .
$$

By Equation (2), and by the geometric interpretation of [Pau, §6], we have

$$
\lambda_{n}=\sum_{i=k_{n}^{\prime}}^{k_{n}^{\prime}+\ell_{n}-1} 2 \operatorname{deg} a_{i}\left(f_{n}\right)=\sum_{i=k_{n}}^{k_{n}+\ell_{n}-1} 2 \operatorname{deg} a_{i}\left(P^{n} f\right) .
$$

With $p_{\infty}: X \rightarrow \Gamma \backslash V \mathbb{T}$ the canonical projection given by $\Gamma g \mapsto \Gamma g *$, we defined in [KePS, Eq. 4] the height of $x \in X$ by

$$
\operatorname{ht}_{\infty}(x)=d_{\Gamma \backslash V \mathbb{T}}\left(p_{\infty}(x), \Gamma *\right) .
$$


Note that $\mathrm{ht}_{\infty}(x a)=\mathrm{ht}_{\infty}(x)$ for all $x \in X$ and $a \in \underline{A}(\mathscr{O})$. Hence using again Equation (2) and the geometric interpretation of [Pau, §6], we have

$$
\begin{aligned}
\mathrm{ht}_{\infty}\left(\Gamma g_{f_{n}} b_{n}\right) & \leq \sup _{i \in \mathbb{Z}} \mathrm{ht}_{\infty}\left(\Gamma g_{f_{n}} b_{n} \mathfrak{a}_{0}^{i}\right)=\sup _{i \in \mathbb{Z}} \mathrm{ht}_{\infty}\left(\Gamma g_{f_{n}} a_{f_{n}} \mathfrak{a}_{0}^{i}\right) \\
& =\max _{i=k_{n}^{\prime}, \ldots, k_{n}^{\prime}+\ell_{n}-1} \operatorname{deg} a_{i}\left(f_{n}\right)=\max _{i=k_{n}, \ldots, k_{n}+\ell_{n}-1} \operatorname{deg} a_{i}\left(P^{n} f\right) .
\end{aligned}
$$

By [KePS, Eq. (14)] and the four lines following it, there exists $c>0$ such that for every $N \in \mathbb{N}$,

$$
\liminf _{n \rightarrow+\infty} \frac{2\left(\max _{i=k_{n}, \ldots, k_{n}+\ell_{n}-1} \operatorname{deg} a_{i}\left(P^{n} f\right)-N\right)}{2 \sum_{i=k_{n}}^{k_{n}+\ell_{n}-1} \operatorname{deg} a_{i}\left(P^{n} f\right)} \geq \liminf _{n \rightarrow+\infty} \frac{2\left(\mathrm{ht}_{\infty}\left(\Gamma g_{f_{n}} b_{n}\right)-N\right)}{\lambda_{n}} \geq c .
$$

This indeed proves the first assertion of Theorem 1.

The second assertion of Theorem 1 follows from [KePS, Lem. 13], whose hypothesis are exactly the ones of this second assertion. The change between lim inf in the definition of $c$-degree-escaping continued fraction expansions and limsup in the second assertion of Theorem 1 comes from the fact that we need in the final Remark of [KePS, §4.1] to take a subsequence of the sequence of vertices along the rational Hecke ray in order to obtain full escape of mass.

In order to prove the last assertion of Theorem 1, let again $P \in R$ be an irreducible polynomial. Since the $P$-Hecke tree of $x=\Gamma g \in X$ is

$$
T_{P}(x)=\left\{g^{-1} \gamma^{-1} \alpha_{0}\left(P^{n}\right)[R \times R]: \gamma \in \Gamma, n \in \mathbb{N}\right\},
$$

since $\alpha_{0}\left(P^{-n}\right) \gamma g_{f}$ and $g_{P^{n} \gamma \cdot f}$ have the same $A$-orbit on the right by Equation (6), Theorem 1 (3) then follows from [KePS, §4.2].

\section{Proof of Theorem 2 in the introduction.}

We will actually prove the stronger statement that the uncountably many sequences $\left(\gamma_{n}^{\prime \prime}\right)_{n \in \mathbb{N}}$ in $\Gamma$ we are going to construct satisfy the fact that for each one of them, there exists an increasing sequence $\left(k_{n}\right)_{n \in \mathbb{N}}$ in $\mathbb{N}$ such that the images of $\gamma_{n}^{\prime \prime}$ and $\gamma_{n+1}^{\prime \prime}$ in the group $\mathrm{PGL}_{2}\left(R /\left(P^{k_{n}} R\right)\right)$, by the reduction modulo $P^{k_{n}}$ of their coefficients, are equal.

Let $P \in R$ be an irreducible polynomial. Let $x \in X$ and $g \in G$ be such that $x=\Gamma g$. We again refer to [KePS, §2.5] for the basic facts we are using on the Hecke trees. For every point at infinity $\xi$ of the Hecke tree $T_{P}(x)$, let $\left(x_{n}^{\xi}\right)_{n \in \mathbb{N}}$ be the sequence of vertices along the geodesic ray in $T_{P}(x)$ from $x$ to $\xi$. In particular $x_{n}^{0}=g^{-1}\left[R \times P^{n} R\right]$. Recall that the action of $\Gamma$ on the sphere of radius $n \in \mathbb{N}$ centered at $x$ in $T_{P}(x)$ is transitive. Hence there exists a sequence $\left(\gamma_{n}\right)_{n \in \mathbb{N}}$ in $\Gamma$ such that $x_{n}^{\xi}=g^{-1} \gamma_{n}^{-1}\left[R \times P^{n} R\right]$. The fact that $x_{n}^{\xi}$ belongs to the geodesic segment from $x$ to $x_{n+1}^{\xi}$ is equivalent to the fact that $\gamma_{n+1} \gamma_{n}^{-1}$ belongs to the mod $P^{n}$-upper triangular subgroup of $\Gamma$, which is the subgroup of elements of $\Gamma$ whose $(2,1)$-coefficient vanishes modulo $P^{n}$.

By [KePS, Theo. 4], there exists $c^{\prime}>0$ and uncountably many points at infinity $\xi$ of $T_{P}(x)$ such that for every $A$-periodic $y \in X$, there exists an increasing sequence $\left(n_{k}\right)_{k \in \mathbb{N}}$ in $\mathbb{N}$ such that the weak-star $\operatorname{limit} \theta=\lim _{k \rightarrow \infty} \mu_{x_{n_{k}}}$ exists and satisfies $\theta \geq c^{\prime} \mu_{y}$. Since $F: M_{A} \rightarrow M_{\mathscr{M}}$ is weak-star continuous, linear and order preserving by Theorem 4 (2), we 
have $\lim _{k \rightarrow \infty} F\left(\mu_{x_{n_{k}}}\right) \geq c^{\prime} F\left(\mu_{y}\right)$. Hence since the measures are finite and nonzero, with $c^{\prime \prime}=\frac{c^{\prime}\left\|F\left(\mu_{y}\right)\right\|}{\|\theta\|}>0$ (which depends on $\xi, y$ and the subsequence), we have

$$
\lim _{k \rightarrow \infty} \frac{F\left(\mu_{x_{n_{k}}^{\xi}}\right)}{\| F\left(\mu_{x_{n_{k}}^{\xi}} \|\right.} \geq c^{\prime \prime} \frac{F\left(\mu_{y}\right)}{\left\|F\left(\mu_{y}\right)\right\|} .
$$

Now let $f \in Q I$. We may assume that $f \in \mathscr{M}^{\natural}$ and $f^{\sigma} \in\left({ }^{c} \mathscr{O}\right)^{\natural}$, up to replacing $f$ by $\gamma \cdot f$ by some $\gamma \in \Gamma$, which does not change the conclusion of Theorem 2. We now take $x=x_{f} \in C$ and $g=g_{f} a_{f}$, so that $x_{f}=\Gamma g_{f} a_{f}$ (see the lines above Lemma 6). We consider the uncountably many sequences $\left(\gamma_{n}\right)_{n \in \mathbb{N}}$ in $\Gamma$ associated with the above uncountably many points at infinity $\xi$ of $T_{P}\left(x_{f}\right)$. Let $\beta_{n} \in \Gamma$ be such that

- $f_{n}=\beta_{n} \cdot\left(P^{n} \gamma_{n} \cdot f\right)$ belongs to $\mathscr{M}^{\natural}$ and $f_{n}{ }^{\sigma} \in\left({ }^{c} \mathscr{O}\right)^{\natural}$,

- and besides, such that $\nu_{f_{n}}=\nu_{P^{n} \gamma_{n} \cdot f}$ (in order to have this, we might need to multiply a $\beta_{n}$ satisfying the first point by an element of $\alpha_{0}\left(\mathbb{F}_{q}^{\times}\right) \subset \Gamma$, see [BN, Theo. 1] for an explanation).

Since $\beta_{n} \alpha_{0}\left(P^{-n}\right) \gamma_{n} \in \mathrm{PGL}_{2}(K)$ and by Equation (5), there exists $b_{n} \in A$ such that

$$
\beta_{n} \alpha_{0}\left(P^{-n}\right) \gamma_{n} g_{f}=g_{\beta_{n} \alpha_{0}\left(P^{-n}\right) \gamma_{n} \cdot f} b_{n}=g_{f_{n}} b_{n} .
$$

Hence

$$
x_{n}^{\xi}=\left(g_{f} a_{f}\right)^{-1} \gamma_{n}^{-1}\left[R \times P^{n} R\right]=\Gamma \alpha_{0}\left(P^{-n}\right) \gamma_{n} g_{f} a_{f}=\Gamma g_{f_{n}} b_{n} a_{f},
$$

which is in the same $A$-orbit as $\Gamma g_{f_{n}} a_{f_{n}}$. By Lemma 6, we have $\Gamma g_{f_{n}} a_{f_{n}} \in C$ and $\Theta_{2}\left(\Gamma g_{f_{n}} a_{f_{n}}\right)=f_{n}$. Hence by Theorem 4 (4), we have

$$
\frac{F\left(\mu_{x_{n}^{\xi}}\right)}{\| F\left(\mu_{x_{n}^{\xi}} \|\right.}=\frac{F\left(\mu_{\Gamma g_{f_{n}} a_{f_{n}}}\right)}{\| F\left(\mu_{\Gamma g_{f_{n}} a_{f_{n}}} \|\right.}=\nu_{f_{n}}=\nu_{P^{n} \gamma_{n} \cdot f} .
$$

Let $f^{\prime} \in Q I$ and $y=x_{f^{\prime}}$, which is $A$-periodic. Up to replacing $f^{\prime}$ by $\gamma \cdot f^{\prime}$ by some $\gamma \in \Gamma$, we assume that $f^{\prime} \in \mathscr{M}$ and $\left(f^{\prime}\right)^{\sigma} \in\left({ }^{c} \mathscr{O}\right)^{\natural}$, so that $\Theta_{2}(y)=f^{\prime}$ again by Lemma 6 . Similarly, by Theorem 4 (4), we have

$$
\frac{F\left(\mu_{y}\right)}{\| F\left(\mu_{y} \|\right.}=\nu_{f^{\prime}}
$$

The result then follows from Equation (8).

\section{References}

[AS] M. Aka and U. Shapira. On the evolution of continued fraction expansions in a fixed quadratic field. Preprint [arXiv:1201.1280], to appear in Journal d'Analyse Mathématique.

[BN] V. Berthé and H. Nakada. On continued fraction expansions in positive characteristic: equivalence relations and some metric properties. Expo. Math. 18 (2000) 257-284.

[BP] A. Broise-Alamichel and F. Paulin. Dynamique sur le rayon modulaire et fractions continues en caractéristique p. J. London Math. Soc. 76 (2007) 399-418. 
[BPP] A. Broise-Alamichel, J. Parkkonen, and F. Paulin. Equidistribution and counting under equilibrium states in negatively curved spaces and graphs of groups. Applications to nonArchimedean Diophantine approximation. Book preprint (318 pages) arXiv:1612.06717.

[BuM] M. Burger and S. Mozes. CAT(-1) spaces, divergence groups and their commensurators. J. Amer. Math. Soc. 9 (1996) 57-94.

[dMT] B. de Mathan and O. Teulié. Problèmes diophantiens simultanés. Monatsh. Math. 143 (2004) 229-245.

[EW] M. Einsiedler and T. Ward. Ergodic theory with a view towards number theory. Grad. Texts Math. 259, Springer-Verlag, 2011.

[KePS] A. Kemarsky, F. Paulin, and U. Shapira. Escape of mass in homogeneous dynamics in positive characteristic. J. Modern Dyn. 11 (2017) 369-407.

[Kit] B. P. Kitchens. Symbolic Dynamics: One-sided, Two-sided and Countable State Markov Shifts. Universitext, Springer, 1998.

[Las] A. Lasjaunias. A survey of Diophantine approximation in fields of power series. Monat. Math. 130 (2000) 211-229.

[Nag] H. Nagao. On GL(2, K[x]). J. Inst. Polytech. Osaka City Univ. Ser. A 10 (1959) 117-121.

[Pau] F. Paulin. Groupe modulaire, fractions continues et approximation diophantienne en caractéristique p. Geom. Dedi. 95 (2002) 65-85.

[Rob] T. Roblin. Ergodicité et équidistribution en courbure négative. Mémoire Soc. Math. France, 95 (2003).

[Sch] W. Schmidt. On continued fractions and diophantine approximation in power series fields. Acta Arith. XCV (2000) 139-166.

[Ser] J.-P. Serre. Arbres, amalgames, $S L_{2}$. 3ème éd. corr., Astérisque 46, Soc. Math. France, 1983.

[Wei] A. Weil. On the analogue of the modular group in characteristic p. In "Functional Analysis and Related Fields" (Chicago, 1968), pp. 211-223, Springer, 1970.

Laboratoire de mathématique d'Orsay,

UMR 8628 Univ. Paris-Sud, CNRS

Université Paris-Saclay, 91405 ORSAY Cedex, FRANCE

e-mail: frederic.paulin@math.u-psud.fr

Mathematics Department, Technion

Israel Institute of Technology, Haifa, 32000 ISRAEL.

e-mail: ushapira@tx.technion.ac.il 